\title{
The single copy of the gravitational holonomy
}

\author{
Rashid Alawadhi, David S. Berman, Chris D. White and Sam Wikeley \\ Centre for Theoretical Physics, Department of Physics and Astronomy, \\ Queen Mary University of London, \\ Mile End Road, London E1 4NS, U.K. \\ E-mail: r.alawadhi@qmul.ac.uk, d.s.berman@qmul.ac.uk, \\ christopher.white@qmul.ac.uk, s.wikeley@qmul.ac.uk
}

ABstract: The double copy is a well-established relationship between gravity and gauge theories. It relates perturbative scattering amplitudes as well as classical solutions, and recently there has been mounting evidence that it also applies to non-perturbative information. In this paper, we consider the holonomy properties of manifolds in gravity and prescribe a single copy of gravitational holonomy that differs from the holonomy in gauge theory. We discuss specific cases and give examples where the single copy holonomy group is reduced. Our results may prove useful in extending the classical double copy. We also clarify previous misconceptions in the literature regarding gravitational Wilson lines and holonomy.

Keywords: Scattering Amplitudes, Classical Theories of Gravity, Wilson, 't Hooft and Polyakov loops

ArXiv EPrint: 2107.01114 


\section{Contents}

1 Introduction 1

2 Holonomy and Wilson lines $\quad 3$

2.1 Riemannian holonomy 3

2.2 Mathematical formulation of holonomy 4

2.3 The spin connection holonomy 5

2.4 Holonomy in gauge theory $\quad 7$

2.5 The gravitational Wilson line 8

3 The single copy of the gravitational holonomy 10

$\begin{array}{lll}3.1 & \text { Relativistic spinning particles } & 10\end{array}$

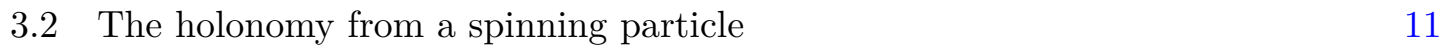

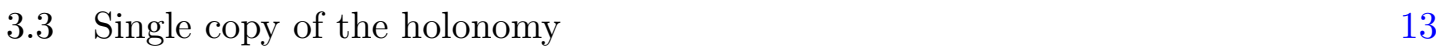

$\begin{array}{ll}3.4 & \text { Relation to scattering amplitudes } \\ & 14\end{array}$

$\begin{array}{ll}3.5 & \text { Insights from Kerr-Schild solutions } \\ \end{array}$

4 Results for the single copy holonomy operator 18

$\begin{array}{ll}4.1 \text { The Schwarzschild black hole } & 19\end{array}$

4.2 Taub-NUT space 21

4.3 Self-dual solutions 25

$\begin{array}{llr}5 & \text { Discussion } & 27\end{array}$

A Derivation of the Kerr-Schild spin connection $\quad 28$

\section{Introduction}

The double copy [1, 2] is by now a well-established relationship between gauge theory and gravity, that has been demonstrated for scattering amplitudes as well as classical solutions. An important focus of ongoing research is to ascertain how general the double copy is, and in particular whether it applies to non-perturbative information. A successful understanding of the latter may elucidate the underlying origin of the double copy, or reveal new ways of thinking about different field theories, that make their common structures manifest.

Previous attempts to study non-perturbative effects include analysing strong coupling solutions of equations of motion [3-7], examining exact algebras underlying the kinematic sectors of different theories [8-10], using twistor methods [11-13], matching solution-generating transformations between different theories [14-17], and studying whether topological information (such as characteristic classes) can be identified in gauge and gravity solutions $[18,19]$ 
(see also ref. [20]). These studies suggest that it is worthwhile to consider other global properties of gauge or gravity solutions, and to ascertain whether or not they can be matched according to a double copy prescription. In this paper, we study the notion of holonomy which, loosely speaking, describes how a vector is transformed after parallel transport around a closed curve. The set of all such transformations forms a holonomy group and particular elements of the holonomy group are described by path ordered integrals of the Christoffel connection along the curve. In gauge theories, the equivalent concept is how the phase of a charged particle transforms as it moves along a path. This is then described by Wilson line operators, which involve integrating the gauge field along a curve.

It has long been known that holonomy properties of gauge and gravity theories are mathematically analogous. Gauge theories can be thought of in terms of principal fibre bundles, where a base space (corresponding to spacetime) is dressed by fibres acted on by the gauge group. The gauge field itself is then associated with a connection on the fibre bundle. The description in gravity is similar: one considers the tangent bundle obtained by dressing spacetime with its tangent space at each point such that the tangent space is now the fibre. The connection on this bundle corresponds to the connection in gravity. Thus, the holonomy in gauge and gravity theories share a common geometrical origin in that they both arise due to the fact that parallel transport in the base manifold induces transport in the fibre. It is therefore tempting to conclude that the holonomy groups of gauge and gravity theories are directly related by the double copy. As we will discuss in detail in this paper, this assumption is false.

Attempts to explicitly relate holonomy properties and/or Wilson lines in gauge and gravity theories have been made before. In particular, refs. [21, 22] studied the holonomy properties of gravity solutions using a perturbative field theory approach, based on Wilson line operators involving the Christoffel symbol. ${ }^{1}$ The behaviour of these operators in perturbation theory was found to be in striking contrast with the situation in gauge theory, an observation that has arisen more recently in the study of perturbative Wilson loops [26]. Given that the double copy relates scattering amplitudes in perturbation theory, this already suggests that the traditional holonomy operator in gravity is not a double copy of its gauge theory counterpart. Indeed, there is a second operator that one may write down in gravity, involving the path-length of a particle traversing a curve, which has also been called a Wilson line [26-30]. It represents the phase experienced by a scalar particle that travels around a closed loop (see also the earlier work of ref. [31]), and in this sense is the correct physical analogue of the holonomy operator in gauge theory, which arises in the description of the Aharonov-Bohm effect. It has also been used in the description of soft radiation [29], and highenergy scattering $[32,33]$, in both cases overlapping with results that can also be obtained via the double copy of scattering amplitudes [34-36]. Recently, ref. [19] argued that the single copy of this second operator is indeed the (unique) gauge theory Wilson line, and that it can be used to express non-trivial topological information of gauge and gravity solutions in a common language (see also ref. [37] for a related study from a different point of view).

\footnotetext{
${ }^{1}$ Similar gravitational Wilson lines have been used in lattice studies of quantum gravity [23, 24], and even date back to much earlier work [25] that attempted to recast General Relativity in a manifestly coordinate-independent form.
} 
The above discussion begs the following question: does the holonomy operator in gravity have a single copy? The aim of this paper is to systematically explore this question, and thus construct a square of four operators, such that we have a pair of gauge theory operators which are a meaningful single copy of the two gravity operators mentioned above. Doing so will allow us to clear up some confusions in the literature regarding the nature of Wilson lines in gravity, as well as provide yet more glimpses of potential non-perturbative aspects of the double copy.

The structure of our paper is as follows. In section 2, we review relevant topics in the study of holonomy and Wilson lines. In section 3, we discuss the single copy of the Riemannian holonomy, as well as its relationship to an alternative holonomy operator involving the spin connection, which will prove useful for what follows. In section 4 , we present explicit results for the single copy operator, performing a detailed comparison with its gravitational counterpart. Finally, we discuss our results and conclude in section 5 .

\section{Holonomy and Wilson lines}

As discussed above, holonomy refers, in general, to the change in certain mathematical objects as they are transported around a closed loop. The particular description depends on which theory we are in, as well as which quantities are being transported. In this section, we review the relevant ideas that we will need for the rest of the paper.

\subsection{Riemannian holonomy}

Given a (pseudo-)Riemannian manifold $\mathcal{M}$, one may consider the tangent space $T_{p}(\mathcal{M})$ of all vectors at a point $p$. The tangent spaces $T_{p}(\mathcal{M})$ and $T_{q}(\mathcal{M})$ associated with points $p, q \in \mathcal{M}$ will be different in general, such that one must define a prescription for comparing vectors at different points. As is well-known, the solution is to consider a curve $\gamma(t)$ from $p$ to $q$, and to say that a given vector $V^{\mu}$ undergoes parallel transport along the curve if it satisfies the equation

$$
\frac{d}{d t} V^{\mu}+\Gamma_{\sigma \rho}^{\mu} \frac{d x^{\rho}}{d t} V^{\sigma}=0
$$

where $\Gamma_{\sigma \rho}^{\mu}$ is the Christoffel symbol. Vectors may be compared after they are paralleltransported to the same point (or tangent space), and one may indeed solve eq. (2.1) to find the total change in $V^{\mu}$ after it has been transported from $p$ to $q$ :

$$
V_{q}^{\mu}=\left[\Phi_{\Gamma}(\gamma)\right]_{\sigma}^{\mu} V_{p}^{\sigma}, \quad\left[\Phi_{\Gamma}(\gamma)\right]_{\sigma}^{\mu}=\mathcal{P} \exp \left[-\int_{\gamma} d x^{\rho} \Gamma_{\rho \sigma}^{\mu}\right] .
$$

Here, in a slight abuse of notation, we have exponentiated the Christoffel symbol considered as the matrix $\left[\Gamma_{\rho}\right]^{\mu}{ }_{\sigma}$. Furthermore, the path-ordering symbol $\mathcal{P}$ indicates that, in expanding the exponential, these matrices are to be ordered according to increasing parameter value $\tau$. A special case of eq. (2.2) occurs if $q$ and $p$ are taken to be the same point, on a closed curve $C$. The vectors appearing on the left- and right-hand sides of eq. (2.2) are then living in the same tangent space, such that the change in $V^{\mu}$ upon being transported around $C$ is effected by the transformation matrix

$$
\left[\Phi_{\Gamma}(C)\right]_{\sigma}^{\mu}=\mathcal{P} \exp \left[-\oint_{C} d x^{\rho} \Gamma_{\rho \sigma}^{\mu}\right]
$$


which we will call the Riemannian holonomy operator. The set of all such transformations forms the Riemannian holonomy group. For a generic $d$-dimensional Riemannian manifold, one expects this group to be the maximal set of possible transformations on vectors in the tangent space, namely $\mathrm{O}(d)$. If the manifold is orientable this group will reduce to $\mathrm{SO}(d)$. Further reductions occur in other special cases [38], the details of which have been of much study in the mathematical literature. For example, the holonomy group reduces to $\mathrm{SU}(d / 2)$ for Calabi-Yau manifolds, to $\operatorname{Sp}(d / 4)$ for hyper-Kähler manifolds, and even to $\mathrm{G}_{2}$ for exceptional seven dimensional manifolds.

For the benefit of a more mathematical audience, it is worthwhile to briefly review how holonomy can be defined in more formal terms, which is the subject of the following section.

\subsection{Mathematical formulation of holonomy}

The approach taken in the mathematics literature is to study the holonomy of a given manifold by examining the parallel structures it contains. More precisely, let $E$ be a vector bundle over a base manifold $M$, and $\nabla$ a connection on $M$. This then defines parallel transport as above. For any piece-wise connected curve $\gamma:[a, b] \subset \mathbb{R} \rightarrow M$ an isomorphism of the vector spaces in the fibre of $E$ is defined as

$$
\tau_{\gamma}: E_{\gamma(a)} \rightarrow E_{\gamma(b)}
$$

To introduce the holonomy group $\operatorname{Hol}(\nabla)$, we fix a point $p \in M$ and parallel transport a section of the bundle $X_{p} \in \Gamma\left(E_{p}\right)$ along all piece-wise connected loops at $p$. This defines the holonomy group of the connection $\operatorname{Hol}_{p}(\nabla)$. If we restrict ourselves to null-homotopic loops (loops that are contractible to a point) then we find the restricted holonomy group $\operatorname{Hol}_{p}^{0}(\nabla)$. If the manifold is simply-connected then $\operatorname{Hol}_{p}^{0}(\nabla)=\operatorname{Hol}_{p}(\nabla)$, with the obvious fundamental group homomorphism $\pi_{1}(M) \rightarrow \operatorname{Hol}_{p}(\nabla) / \operatorname{Hol}_{p}^{0}(\nabla)$. Since the holonomy groups of a connected manifold at different points are isomorphic, we can talk about the holonomy group of the connection $\operatorname{Hol}(\nabla) \subseteq \mathrm{GL}(d, E)$.

A section $X \in \Gamma(E)$ is called parallel if it is constant with respect to the connection, i.e. $\nabla X=0$. This is equivalent to saying that $X$ is invariant under the parallel transport map

$$
\tau_{\gamma}: X_{\gamma(a)} \rightarrow X_{\gamma(b)}
$$

for any piece-wise connected path $\gamma:[a, b] \rightarrow M$. Equivalently, we may write $\tau_{\gamma}\left(X_{\gamma(a)}\right)=$ $X_{\gamma(b)}$, which is the non-coordinate basis expression of eq. (2.2). Let us state the fundamental principle [39]:

Theorem. There exists a one-to-one correspondence between parallel sections $X$ of the bundle $E$ and vectors $X_{p}$ in the fibre $E_{p}$ invariant under $\operatorname{Hol}_{\mathrm{p}}(\nabla)$.

In other words, finding on a given (pseudo-)Riemannian manifold geometric objects whose covariant derivative vanishes is equivalent to finding the invariants of the holonomy group [40]. To elucidate the above statements, we now consider some examples of common Riemannian manifolds. 
Example 1. On orientable Riemannian manifolds one can define the metric tensor $g$ as a section on the tensor product of the dual tangent bundle with itself $g \in \Gamma\left(T M^{*} \otimes T M^{*}\right)$, so that the metric tensor at a point $p \in M$ is a map $g_{p}: T_{p} M \otimes T_{p} M \rightarrow \mathbb{R}$. There is a unique torsion-less connection called the Levi-Civita connection such that $\nabla g=0$. Therefore, $g$ is called parallel. To find $\operatorname{Hol}_{p}(\nabla)$, denote the group of linear transformations preserving $g$ by $\mathrm{O}\left(T_{p} M, g_{p}\right)$. Since $g$ is parallel, we find $\operatorname{Hol}_{p}(\nabla) \subset \mathrm{O}\left(T_{p} M, g_{p}\right)$. Furthermore, as $T_{p} M \cong \mathbb{R}^{d}$ we identify the holonomy group with a subgroup in $\mathrm{SO}(d)$.

Example 2. Kähler Manifolds are complex manifolds of dimension $d=2 n$ and have a closed symplectic form $\omega=g(J, \cdot) \in \Lambda^{2}\left(M_{\mathbb{C}}\right)$, where $J$ is the almost complex structure and $\nabla \omega=0$. Now not only the metric tensor has vanishing exterior covariant; the symplectic form $\omega$, which is invariant under the symplectic group $\operatorname{Sp}(2 n, \mathbb{R})$, also has vanishing covariant derivative. Going by our philosophy, the holonomy group must both preserve lengths and the symplectic form. The sought after group is then $\mathrm{U}(n)$. This can be seen as the intersection

$$
\mathrm{U}(n)=\mathrm{SO}(2 n) \cap \mathrm{Sp}(2 n, \mathbb{R}) .
$$

Example 3. Hyper-Kähler manifolds of dimension $d=4 n$ have three almost complex structures $(I, J, K)$ with vanishing covariant derivative and obey the quaternionic relations $I^{2}=J^{2}=K^{2}=I d$ and $I J=J I=-K$. The subgroup of $\mathrm{SO}(4 n)$ that preserves the quaternionic almost complex structure is $\operatorname{Sp}(n) \subset \mathrm{SO}(4 n)$. Therefore $\operatorname{Hol}(\nabla)=\operatorname{Sp}(n)$. An example of this is the holonomy group of the self-dual Taub-NUT metric with $\operatorname{Hol}(\nabla)=$ $\mathrm{Sp}(1) \cong \mathrm{SU}(2)$.

\subsection{The spin connection holonomy}

In eq. (2.1) we have defined parallel transport explicitly with the Christoffel connection. One may also use an alternative formalism involving the spin connection, which would in any case be necessary if one were to consider the parallel transport of spinors. Let us now describe the role of the spin connection. As is well known (see e.g. ref. [41] for a pedagogical summary), at each point in spacetime, one may introduce a set of orthonormal basis vectors $\hat{e}_{(a)}$, related to the usual tangent space vector basis $e_{(\mu)} \equiv \partial_{\mu}$ by

$$
e_{(\mu)}=e_{\mu}^{a} \hat{e}_{(a)}, \quad \hat{e}_{(a)}=e_{a}^{\mu} e_{(\mu)}
$$

which defines the (inverse) vielbein $e^{a}{ }_{\mu}\left(e_{a}{ }^{\mu}\right)$, satisfying

$$
e^{a}{ }_{\mu} e^{b}{ }_{\nu} \eta_{a b}=g_{\mu \nu}, \quad e_{a}^{\mu} e_{b}^{\nu} g_{\mu \nu}=\eta_{a b}, \quad e^{a}{ }_{\mu} e_{b}^{\mu}=\delta_{b}^{a}, \quad e^{a}{ }_{\mu} e_{a}^{\nu}=\delta_{\mu}^{\nu},
$$

where $\eta_{a b}$ is the Minkowski metric of the flat tangent space. ${ }^{2}$ Thus, the Roman indices $(a, b, \ldots)$ are raised and lowered with this flat metric, while the Greek indices $(\mu, \nu, \ldots)$ are raised and lowered using the curved metric $g_{\mu \nu}$.

The vielbein may now be used to relate the components of an arbitrary vector $V^{\mu}$ at a point $p$ to the components of a vector in the tangent space at $p$ via

$$
V^{a}=e^{a}{ }_{\mu}(p) V^{\mu}, \quad V^{\mu}=e_{a}^{\mu}(p) V^{a} .
$$

\footnotetext{
${ }^{2}$ We use a $(-,+,+,+)$ metric signature throughout.
} 
From the vielbein we may calculate the spin connection $\omega_{b}^{a}$ using Cartan's first structure equation. In differential form language, with the torsion set to zero, this is:

$$
d e^{a}+\omega_{b}^{a} \wedge e^{b}=0 .
$$

We may also invert this to write the components of the spin connection in terms of the vielbein:

$$
\left(\omega_{\mu}\right)^{a b}=\frac{1}{2} e^{a \nu}\left(\partial_{\mu} e^{b}{ }_{\nu}-\partial_{\nu} e^{b}{ }_{\mu}\right)-\frac{1}{2} e^{b \nu}\left(\partial_{\mu} e^{a}{ }_{\nu}-\partial_{\nu} e^{a}{ }_{\mu}\right)-\frac{1}{2} e^{a \rho} e^{b \sigma} e_{\mu}^{c}\left(\partial_{\rho} e_{c \sigma}-\partial_{\sigma} e_{c \rho}\right) .
$$

A vector in the tangent space will then satisfy the parallel transport equation as in eq. (2.2), but now with the spin connection:

$$
\frac{d}{d t} V^{a}+\left(\omega_{\mu}\right)_{b}^{a} \frac{d x^{\mu}}{d t} V^{b}=0
$$

The solution of eq. (2.12), by direct analogy with eq. (2.2), is

$$
V_{q}^{a}=\left[\Phi_{\omega}(\gamma)\right]_{b}^{a} V_{p}^{b}, \quad\left[\Phi_{\omega}(\gamma)\right]_{b}^{a}=\mathcal{P} \exp \left[-\int_{\gamma} d x^{\mu}\left(\omega_{\mu}\right)^{a}{ }_{b}\right],
$$

where $\mathcal{P}$ denotes path-ordering of the matrices $\left(\omega_{\mu}\right)^{a}{ }_{b}$ along the worldline. Choosing $p$ and $q$ to correspond to the same point on a closed curve $C$, one obtains the holonomy of the spin connection:

$$
\left[\Phi_{\omega}(C)\right]_{b}^{a}=\mathcal{P} \exp \left[-\oint_{C} d x^{\mu}\left(\omega_{\mu}\right)_{b}^{a}\right] .
$$

This form of the holonomy is straightforwardly related to the Riemannian holonomy operator of eq. (2.3). Upon transforming both sides of eq. (2.2) to the orthonormal basis and rearranging, one obtains [22]

$$
\left[\Phi_{\omega}(p, q)\right]_{b}^{a}=e_{\mu}^{a}(p)\left[\Phi_{\Gamma}(p, q)\right]_{\nu}^{\mu} e_{b}^{\nu}(q)
$$

so that for a closed curve one has

$$
\left[\Phi_{\omega}(C)\right]_{b}^{a}=e_{\mu}^{a}\left[\Phi_{\Gamma}(C)\right]_{\nu}^{\mu} e_{b}{ }^{\nu},
$$

where the two vielbeins on the right-hand side are evaluated at the same point. The physical interpretation of this relation is straightforward. The Riemannian holonomy operator tells us how the components of a vector transform after the vector has been transported around $C$. The expression in terms of the spin connection does the same, but in the orthonormal basis. Then, the two holonomy operators are related by a similarity transformation, which is the content of eq. (2.16).

Thus, in discussing the holonomy of a given manifold, one is free to use either. For our later purposes, it is convenient to rewrite eq. (2.14) yet further. Noting that the spin connection is valued in the Lie algebra of the Lorentz group, we may introduce explicit Lorentz generators $M^{a b}$ via

$$
\left(\omega_{\mu}\right)_{d}^{c}=\frac{i}{2}\left(\omega_{\mu}\right)_{a b}\left(M^{a b}\right)_{d}^{c}
$$


where the normalisation factor arises from the components of the generators in the spin-1 representation:

$$
\left(M^{a b}\right)_{d}^{c}=i\left(\eta^{a c} \delta_{d}^{b}-\eta^{b c} \delta_{d}^{a}\right) .
$$

The operator of eq. (2.14) is then

$$
\left[\Phi_{\omega}(C)\right]_{d}^{c}=\mathcal{P} \exp \left[-\frac{i}{2} \oint_{C} d x^{\mu}\left(\omega_{\mu}\right)_{a b} M^{a b}\right]_{d}^{c} .
$$

Here it is worth noting that this form allows us to easily extend the notion of holonomy to spinors. One replaces the generators in the spin- 1 representation with the generators in the spin- $(1 / 2)$ representation constructed from the associated Clifford algebra. Concretely, instead of $M^{a b}$ one uses $\left(\Gamma^{a b}\right)^{\alpha}{ }_{\beta}$ and the holonomy group will be generically $\operatorname{Spin}(d)$ valued. Doing this relies on lifting the tangent bundle of the manifold to a spin bundle, which requires the existence of a spin structure on the manifold. There may of course be global obstructions to doing this which are given by the second Stiefel-Whitney class. In what follows we will not worry about such spinor-valued holonomies, however it is certainly worth understanding how the double copy works in this case and how different representations in the tangent bundle are related to representations in the single copy. Having described how holonomy works in gravity, let us now consider gauge theory.

\subsection{Holonomy in gauge theory}

Consider a gauge theory defined on a spacetime manifold $\mathcal{M}$ with gauge group $G$. A field $\Psi^{a}$ transforming in a particular representation of the gauge group can then be defined as a section of a principal fibre bundle, where the gauge field itself is associated with the connection. If we want to compare field values at different points $p, q \in \mathcal{M}$, we must transform the gauge information according to a suitable definition of parallel transport, leading to an equation analogous to eq. (2.2):

$$
\Psi_{q}^{a}=\left[\Phi_{A}(\gamma)\right]_{b}^{a} \Psi_{p}^{b}, \quad\left[\Phi_{A}(\gamma)\right]_{b}^{a}=\mathcal{P} \exp \left[-g \int_{\gamma} d x^{\mu} \mathbf{A}_{\mu}\right]_{b}^{a}
$$

Here $\mathbf{A}_{\mu}=A_{\mu}^{a} \mathbf{T}^{a}$ is the matrix-valued gauge field, $\mathbf{T}^{a}$ are the generators of the Lie algebra in the representation appropriate to the field $\Psi^{a}$, and $g$ is the coupling. If we again take $p$ and $q$ to be the same spacetime point lying on a closed curve $C$, the change in $\Psi^{a}$ after transport around the loop is given by

$$
\Phi_{A}(C)=\mathcal{P} \exp \left[-g \oint_{C} d x^{\mu} \mathbf{A}_{\mu}\right] .
$$

The set of all such transformations forms the holonomy group associated with gauge theory solutions, which will be a subgroup of the gauge group $G$.

The operator appearing in eq. (2.20) is known as a Wilson line in the gauge theory literature. ${ }^{3}$ It transforms covariantly under gauge transformations according to

$$
\Phi_{A}(\gamma) \rightarrow \mathbf{U}_{p} \Phi_{A}(\gamma) \mathbf{U}_{q}^{-1},
$$

\footnotetext{
${ }^{3}$ Note that we have chosen anti-Hermitian colour generators, such that there is no explicit factor of $i$ in the exponent of eq. (2.20).
} 
where $\mathbf{U}_{p}$ is an element of $G$ in the appropriate representation, and represents a local gauge transformation at the point $p$. Thus, Wilson lines are ubiquitous in the study of scattering amplitudes, and typically crop up whenever some physical behaviour has to be expressed in a gauge-covariant manner. The operator of eq. (2.21) is known as a Wilson loop once the trace is taken on the right-hand side. From eq. (2.22), this renders the Wilson loop gauge-invariant.

Notably, the gravitational operator of eq. (2.2) also transforms similarly to eq. (2.22), but where the gauge transformations are replaced by diffeomorphisms. That is, upon changing coordinates according to $x^{\alpha} \rightarrow y^{\alpha}$, one has [22]

$$
\left[\Phi_{\Gamma}(\gamma)\right]_{\sigma}^{\mu} \rightarrow\left[\Lambda_{p}\right]_{\alpha}^{\mu}\left[\Phi_{\Gamma}\left(\gamma^{\prime}\right)\right]_{\beta}^{\alpha}\left[\Lambda_{q}^{-1}\right]_{\sigma}^{\beta}, \quad\left[\Lambda_{p}\right]^{\lambda}=\left(\frac{\partial y^{\lambda}}{\partial x^{\delta}}\right)_{p},
$$

where the path $\gamma$ is transformed according to the diffeomorphism to $\gamma^{\prime}$. This property, together with the fact that there is a common geometric interpretation of the operators $\Phi_{\Gamma}(\gamma)$ and $\Phi_{A}(\gamma)$ in gravity and gauge theory respectively, has led to $\Phi_{\Gamma}$ also being referred to as a Wilson line in the gravity literature [21-24]. However, there is a more sensible candidate for this, as we discuss in the following section.

\subsection{The gravitational Wilson line}

In an abelian gauge theory, the Wilson line operator of eq. (2.21) has a useful physical interpretation, in that it represents the phase change experienced by a charged particle as it traverses a closed loop. The non-abelian version is a generalisation of this, once the trace is taken to form a gauge-invariant quantity. The analogous operator in gravity is easy to write down. In gravity the equivalent of the charge is the mass of the particle and so the phase will only depend on the (Lorentz-invariant) path length of the closed curve multiplied by the particle mass. For an arbitrary curve $\gamma$, one may then define the gravitational Wilson line [27] as follows (this is also discussed in the much earlier work of ref. [31]):

$$
\Phi_{g}(\gamma)=\exp \left[-i m \int_{\gamma} d \tau \sqrt{-g_{\mu \nu} \dot{x}^{\mu} \dot{x}^{\nu}}\right]
$$

where $m$ is the mass of the particle, $\tau$ its proper time, and $\dot{x}^{\mu} \equiv d x^{\mu} / d \tau$. Throughout this paper $\dot{x}^{\mu}$ will always denote differentiation with respect to the variable parameterising the curve. In perturbation theory (as appropriate to the weak field limit), one may introduce a graviton field $h_{\mu \nu}$ via

$$
g_{\mu \nu}=\eta_{\mu \nu}+\kappa h_{\mu \nu}, \quad \kappa=\sqrt{32 \pi G_{N}},
$$

where $\eta_{\mu \nu}$ is the Minkowski metric, and $G_{N}$ Newton's constant. Then the operator of eq. (2.24) simplifies, to first non-trivial order in $\kappa$, to $^{4}$

$$
\Phi_{g}(\gamma)=\exp \left[\frac{i \kappa}{2} \int_{\gamma} d s h_{\mu \nu} \dot{x}^{\mu} \dot{x}^{\nu}\right]
$$

\footnotetext{
${ }^{4}$ We have ignored an overall multiplicative constant in eq. (2.26), which will vanish in any vacuum expectation value of Wilson lines, once this is correctly normalised.
} 
where we have rescaled the integration variable to have mass dimension -2 . The square root in eq. (2.24) is cumbersome in general, and one may further worry that the operator ceases to be defined for massless particles. One may remove both problems by noting that the exponent of eq. (2.24) contains the action for a point particle. The latter can be replaced with the alternative action ${ }^{5}$

$$
S_{\mathrm{pp}}=\frac{1}{2} \int d \tau\left[\frac{1}{e(\tau)} g_{\mu \nu} \dot{x}^{\mu} \dot{x}^{\nu}-e(\tau) m^{2}\right]
$$

where $e(\tau)$ is an auxiliary parameter known as the einbein. Its field equation yields

$$
\frac{\delta S}{\delta e}=-\frac{1}{2 e^{2}} g_{\mu \nu} \dot{x}^{\mu} \dot{x}^{\nu}-\frac{m^{2}}{2}=0,
$$

such that solving for $e(\tau)$ and substituting this into eq. (2.27) yields the original action of a point particle that appears in eq. (2.24), in the massive case. The parameter $e(\tau)$ plays the role of a "metric" on the worldline, and transforms appropriately under reparametrizations. Choosing a value for $e$ then amounts to fixing a gauge, and the choice $e=1$ in the massless case immediately leads to the Wilson line of eq. (2.26).

It has recently been argued [19] that the operator $\Phi_{g}(\gamma)$ is the double copy of the gauge theory Wilson line of eq. (2.20), which can be seen in a number of ways. It may be related to scattering amplitudes, for example, by considering a semi-infinite set of Wilson lines emanating from a common point. Vacuum expectation values of such Wilson lines are known to describe the infrared singularities of scattering amplitudes, where the latter have been proven to formally double copy [34]. That eq. (2.24) is the correct Wilson line associated with IR singularities has been established in refs. [28, 29]. Similar evidence comes from the high energy (Regge) limit, where amplitudes are again known to double-copy [35, 36], and where there is also a description in terms of the Wilson line operators of eqs. (2.20) and $(2.26)[32,33,42]$. More directly, one may rewrite the operator in eq. (2.20) as

$$
\Phi(\gamma)=\mathcal{P} \exp \left[i g \tilde{\mathbf{T}}^{a} \int_{\gamma} d s A_{\mu}^{a} \dot{x}^{\mu}\right],
$$

where we have temporarily adopted Hermitian colour generators defined via $\tilde{\mathbf{T}}^{a} \equiv i \mathbf{T}^{a}$. If we consider a gauge field for which the double copy is known, then the gravitational Wilson line of eq. (2.26) is obtained by making the replacements

$$
g \rightarrow \frac{\kappa}{2}, \quad \tilde{\mathbf{T}}^{a} \rightarrow \dot{x}^{\mu},
$$

precisely mirroring the usual coupling and colour/kinematic replacements associated with the BCJ double copy for amplitudes. Note that in the second replacement, the adjoint index $a$ is replaced by a spacetime index $\mu$, in accordance with the fact that colour information is stripped off and replaced by kinematic information when performing the double copy. Further to the discussion in ref. [19], it is interesting to note that the explicit double copy between the gauge and gravity Wilson lines is manifest when using the alternative

\footnotetext{
${ }^{5}$ The use of this alternative point particle action in a double copy context has been emphasised previously in ref. [37].
} 
point particle action of eq. (2.27), for a particular choice of einbein. This is perhaps not surprising: the original BCJ double copy for amplitudes is known to be manifest only in certain generalised gauges, where conventional gauge transformations as well as field redefinitions have potentially been applied. The same property occurs also for classical solutions in general. Thus, the fact that a particular einbein is needed to get the double copy to work - itself a choice of gauge, as described above - is entirely consistent with previous instances of the double copy.

Aside from special kinematic limits of amplitudes, there are also other situations in which $\Phi_{g}(\gamma)$ manifests itself as the double copy of $\Phi_{A}(\gamma)$. It may be used, for example, to quantify certain topological information that is the relevant gravitational counterpart of that obtained in a gauge theory [19]. Furthermore, a particular Wilson loop involving $\Phi_{A}$ may be used to derive the Coulomb potential between two static charges, such that replacing $\Phi_{A}$ with $\Phi_{g}$ instead yields Newton's law of gravity [27]. That the two potentials should indeed be related follows from the non-relativistic limit of the classical double copy between the point charge and the Schwarzschild black hole [43].

Given that the holonomy operator in gauge theory is related to the Wilson line of eq. (2.26) in gravity, it is therefore not true that the holonomy operators in the two theories are related by the double copy. The question then arises of whether one may find a gauge theory single copy of the gravitational holonomy operator of eq. (2.3). Indeed one can, as we explain in the following section.

\section{The single copy of the gravitational holonomy}

Above, we have seen that the holonomy operators in gauge and gravity theory, whilst natural mathematical counterparts of each other, are not physical counterparts in the sense of being related by the double/single copy. To find the correct single copy of the gravity result, one must map the latter to a physical situation whose single copy is already well-known. In the present case, the holonomy operator of eq. (2.19) turns out to arise in the dynamics of spinning particles, whose properties we review in the following section.

\subsection{Relativistic spinning particles}

In eq. (2.27), we have seen the action for a spinless point particle coupled to gravity. It is possible to generalise this to the case in which a (possibly extended) object has an intrinsic angular momentum (see ref. [44] for a modern pedagogical review, and also the classic works of refs. [45-48]). To this end, it is conventional to define a vierbein on the worldline, $e^{A}{ }_{\mu}(\tau)$. The upper-case latin indices $(A, B, \ldots)$ are those of a body-fixed frame; a frame fixed to the point-particle as it traverses the worldline. The vierbein $e^{A}{ }_{\mu}$ therefore relates the body-fixed frame to the general coordinate frame. The angular velocity of the object is then defined to be

$$
\Omega_{\mu \nu}=e_{A \mu} \frac{D e^{A}{ }_{\nu}}{D \tau}
$$

where

$$
\frac{D e^{A}{ }_{\nu}}{D \tau} \equiv \dot{x}^{\alpha} D_{\alpha} e^{A}{ }_{\nu}=\dot{x}^{\alpha}\left(\partial_{\alpha} e^{A}{ }_{\nu}-\Gamma_{\alpha \nu}^{\lambda} e_{\lambda}^{A}\right)
$$


is the spacetime covariant derivative of the body-fixed vierbein. That eq. (3.1) satisfies the expected antisymmetry, $\Omega_{\mu \nu}=-\Omega_{\nu \mu}$, follows from eq. (2.8) and the vanishing of the covariant derivative of the metric tensor $g_{\mu \nu}$. The total action for the object can be written as

$$
S_{\mathrm{tot}}=S_{\mathrm{pp}}+S_{\mathrm{spin}}
$$

where $S_{\mathrm{pp}}$ is the spinless action of eq. (2.27), reflecting the fact that an extended object looks pointlike from a sufficient distance. The correction term due to the spin is ${ }^{6}$

$$
S_{\text {spin }}=\int d \tau\left[\frac{1}{2} \Omega_{\mu \nu} S^{\mu \nu}\right]
$$

where $S^{\mu \nu}$ is the spin tensor of the object, namely the dynamical variable conjugate to the angular velocity. Physically this represents the intrinsic angular momentum of the object (in either a classical or quantum setting).

In general, the vierbein $e^{a}{ }_{\mu}$ that we choose for a given spacetime will not correspond to the body-fixed vierbein $e^{A}{ }_{\mu}$, and there will therefore be a Lorentz transformation that relates the two:

$$
e_{\mu}^{A}=\Lambda_{a}^{A} e^{a}{ }_{\mu}
$$

The combination of terms appearing in eq. (3.4) can then be decomposed as [49]

$$
\begin{aligned}
S_{\mu \nu} \Omega^{\mu \nu} & =S_{\mu \nu} \Lambda_{A}{ }^{a} e_{a}{ }^{\mu} \frac{D \Lambda^{A b} e_{b}{ }^{\nu}}{D \tau} \\
& =S_{a b}\left(\Lambda_{A}{ }^{a} \dot{\Lambda}^{A b}-\left(\omega_{\mu}\right)^{a b} \dot{x}^{\mu}\right),
\end{aligned}
$$

where in the second line we have used eq. (3.2) together with the known relation between the Christoffel symbol and spin connection (see e.g. [41])

$$
\Gamma_{\mu \nu}^{\sigma}=e_{a}^{\sigma} e_{\nu}^{b}\left(\omega_{\mu}\right)_{b}^{a}+e_{a}^{\sigma} \partial_{\mu} e^{a}{ }_{\nu}
$$

\subsection{The holonomy from a spinning particle}

We now argue that the dynamics of spinning particles can be used to construct a physical manifestation of the holonomy operator of eq. (2.19). To this end, note that the two terms in eq. (3.6) have a straightforward physical interpretation: the action of eq. (3.4) dictates the dynamics of the internal spin of the object under consideration, namely how the body-fixed vierbein changes as one proceeds along the worldline. Put another way, the action governs how a vector fixed to the moving object will be modified, and there are clearly two distinct effects causing such a vector to change: (i) the rotation of the object; (ii) the fact that the body-fixed frame is changing due to the underlying spacetime. These two effects are captured by the first and second terms in the second line of eq. (3.6) respectively, where the first (rotation) term would be present even if the object were moving in flat space.

\footnotetext{
${ }^{6}$ In writing eq. (3.4), we have ignored additional gauge-fixing terms which are needed to eliminate residual arbitrary degrees of freedom in the precise definition of the spin tensor. Such terms will not matter for the arguments presented here.
} 
In the previous section, we noted that the point particle action of eq. (2.27) could be used to form a Wilson line operator representing the phase experienced by a particle as it traverses a given contour. To do this, one forms the combination

$$
e^{i S_{\mathrm{pp}}}
$$

and discards terms associated with flat space only i.e. that do not involve the gravitational field. Such terms amount to an overall multiplicative factor, that vanishes upon normalising vacuum expectation values of Wilson lines. It is straightforward to repeat this procedure for the action of eq. (3.3), where the corresponding Wilson line now represents the phase experienced by a spinning particle. A given spin tensor will have the form

$$
S^{a b}(\tau)=Q_{c d}^{a b}(\tau) M^{c d},
$$

where, as above, $\left\{M^{c d}\right\}$ are the Lorentz generators. The quantity $Q_{c d}^{a b}$ denotes how much of each spin generator is "turned on", and may in general depend on the parameter $\tau$ along the worldline. We wish to examine how all possible vectors are transported around all possible loops. Thus, we may choose a spin tensor such that

$$
Q_{c d}^{a b}=\frac{1}{2}\left(\delta_{c}^{a} \delta_{d}^{b}-\delta_{c}^{b} \delta_{d}^{a}\right)
$$

which physically amounts to a democratic assignment of unit spin along all axes. This discussion holds for a classical particle. For a quantum particle in state $|\psi\rangle$, the spin tensor will be given by a normalised expectation value

$$
S^{a b}=\frac{\left\langle\psi\left|Q_{c d}^{a b} M^{c d}\right| \psi\right\rangle}{\langle\psi \mid \psi\rangle} .
$$

However, one may again make the choice of eq. (3.10), and for concreteness we focus on a spin-1 particle with arbitrary orientation. Furthermore, we will take our generalised Wilson line to be matrix-valued in spin space, such that it describes how the spin state of a test particle changes as it moves along its worldline. This is directly analogous to how the gauge theory Wilson line of eq. (2.21) is matrix-valued in colour space, and in practical terms amounts to the replacement

$$
S^{a b} \rightarrow M^{a b}
$$

whilst defining the Wilson line according to the appropriate generalisation of eq. (3.8). The result is

$$
\Phi_{g}^{\text {spin }}(\gamma)=\mathcal{P} \exp \left[\frac{i \kappa}{2} \int_{\gamma} d s\left(h_{\mu \nu} \dot{x}^{\mu} \dot{x}^{\nu}-\dot{x}^{\mu}\left(\omega_{\mu}\right)_{c d} M^{c d}\right)\right],
$$

where the path ordering is now necessary due to the matrix-valued nature of the spin generators in the second term (note that there is also an implicit identity matrix in spin space in the first term). The first term of eq. (3.13) represents how the mass of the test particle couples to gravity, while the second term describes how its spin degrees of freedom couple to gravity. In line with the above remarks, only the second term on the right-hand side of eq. (3.6) is relevant, given that it is this that represents the coupling to the gravitational field. 
As discussed above, a number of previous studies have attempted to identify the operator of eq. (2.3) (and, by association, the operator of eq. (2.19)) as a gravitational Wilson line, due to its mathematical similarity to the Wilson line in gauge theory. However, the double copy tells us that eq. (2.19) is not the gravitational counterpart to the gauge theory Wilson line of eq. (2.21). Rather, it is a spin-dependent correction ${ }^{7}$ to the gravitational Wilson line of eq. (2.26), and represents the additional phase change that a particle experiences if it happens to be spinning. It is interesting to note that a related observation was made as early as the 1960s [31] (see also ref. [25]), predating the introduction of Wilson lines!

\subsection{Single copy of the holonomy}

In the previous section, we have seen that a Wilson line constructed from the action for a spinning particle coupled to gravity contains the holonomy operator of eq. (2.19). This immediately tells us how to take the single copy of the holonomy: we can simply write down the action for a spinning particle coupled to a gauge field, and use this to create a generalised Wilson line that contains a spin correction to the phase. The relevant action for a spinning particle coupled to a gauge field is (see e.g. ref. [50])

$$
S_{\text {gauge }}=\int d \tau\left[\frac{1}{2 e(\tau)} \eta_{\mu \nu} \dot{x}^{\mu} \dot{x}^{\nu}-\frac{e(\tau) m^{2}}{2}+\frac{1}{2} \Omega_{\mu \nu} S^{\mu \nu}+g c^{a}(\tau)\left(\dot{x}^{\mu} A_{\mu}^{a}-\frac{e(\tau)}{2} F_{\mu \nu}^{a} S^{\mu \nu}\right)\right]
$$

Here the first two terms are the usual point particle action of eq. (2.27) considered in Minkowski space. Furthermore, $\Omega_{\mu \nu}$ is the flat space version of the angular velocity of eq. (3.1), such that its contraction with the spin tensor $S^{\mu \nu}$ matches the first term in the second line of eq. (3.6). There are then two terms involving the gauge field, where $c^{a}(\tau)$ is a colour vector obtained by evaluating the expectation value of the colour generator $\tilde{\mathbf{T}}^{a}$ at a given position on the worldline. The first of these terms gives rise to the Wilson line operator of eq. (2.21), once one replaces the expectation value of the colour generator by the generator itself. The remaining term couples the field strength $F_{\mu \nu}^{a}$ to the spin tensor, and thus represents the spin-dependent correction to the vacuum dynamics of the spinning particle due to the presence of a gauge field. This is the precise gauge theory analogue of the spin connection term in eq. (3.6), which amounts to the extra contribution to the spin dynamics of the object arising from the gravitational field.

Indeed, the double copy relationship between gauge theory and gravity actions for spinning particles has been addressed in detail in refs. [50, 51] (see also refs. [52, 53]), which considered radiation from such a particle interacting with a Yang-Mills field. The authors calculated the effects of this radiation perturbatively, before double-copying the results order-by-order in perturbation theory. The resulting system is that of a spinning particle interacting with a graviton, axion and dilaton, which is the usual spectrum arising from the double copy of pure Yang-Mills theory. Thus, roughly speaking, the final term in eq. (3.14) double copies to multiple operators, representing the coupling of the spin to the

\footnotetext{
${ }^{7}$ Physically, the effects of the spin term are suppressed by a power of the emitted graviton momentum, as we discuss in section 3.4. So it is in this sense a small correction to the spinless term in an appropriate kinematic limit.
} 
full field spectrum in the gravity theory. This one-to-many nature of the double copy does not affect our arguments here: given we are taking the single copy, which is many-to-one, we can unambiguously identify the single copy of the graviton spin coupling as the final term appearing in eq. (3.14). Interestingly, refs. $[50,51]$ found that the spinning particle actions in gauge theory and gravity were only strict physical double copies of each other (in the sense that a conserved energy-momentum tensor was found in the gravity theory) if a certain fixed numerical coefficient was placed in front of the spin coupling in the gauge theory (see ref. [52] for a similar conclusion). We shall ignore this complication here, given that such a coefficient is irrelevant in elucidating the group of transformations induced by the generalised Wilson line in the gauge theory. Replacing the spin tensor according to eq. (3.12) as before, as well as fixing the einbein $e=1$, this Wilson line is found to be

$$
\Phi_{\text {spin }}(\gamma)=\mathcal{P} \exp \left[i g \tilde{\mathbf{T}}^{a} \int_{\gamma} d s\left(A_{\mu}^{a} \dot{x}^{\mu}-\frac{1}{2} F_{\mu \nu}^{a} M^{\mu \nu}\right)\right] .
$$

The terms in this operator are obtained from the final two terms in eq. (3.14), namely those explicitly containing the coupling of the worldline to the gauge field. Evaluating the spin correction over a closed curve $C$ gives the single copy of the gravitational holonomy:

$$
\Phi_{F}(C)=\mathcal{P} \exp \left[-\frac{i g}{2} \tilde{\mathbf{T}}^{a} \oint_{C} d s F_{\mu \nu}^{a} M^{\mu \nu}\right] .
$$

As in the gravity theory, we can furnish the operator appearing in the action of eq. (3.14) with a physical interpretation, where we may focus on the case of an abelian gauge theory for simplicity. Identifying the electric and magnetic fields by

$$
F_{0 i}=E_{i}, \quad F_{i j}=\epsilon_{i j k} B_{k},
$$

one finds

$$
\begin{aligned}
F_{\mu \nu} S^{\mu \nu} & =2 F_{0 i} S^{0 i}+F_{i j} S^{i j} \\
& =-2(\boldsymbol{E} \cdot \boldsymbol{d}+\boldsymbol{B} \cdot \boldsymbol{\mu}),
\end{aligned}
$$

where we have defined

$$
d_{i}=-S_{0 i}, \quad \mu_{i}=-\frac{1}{2} \epsilon_{i j k} S^{j k}
$$

Equation (3.18) constitutes the electromagnetic coupling of an electric dipole moment $\boldsymbol{d}$ and magnetic dipole moment $\boldsymbol{\mu}$, and we may consider our test particle to have both of these turned on in general.

\subsection{Relation to scattering amplitudes}

In the previous section, we have used known results from the classical double copy to identify the single copy of the gravitational holonomy. We may also note, however, that our results can be linked to known properties of scattering amplitudes. For example, ref. [54] recently argued that the Kerr (spinning) black hole and its single copy can be obtained from 3-point amplitudes that are double-copies of each other. The implications of this were 
explored further in refs. [55, 56]. Similar conclusions have been obtained independently in refs. [57-59], which also linked the double copy for spinning particles with the well-known (next-to-) soft theorems for emission of low-momentum radiation [29,60-66]. We can see this directly from the above results as follows. Starting with the Riemannian parallel transport operator of eq. (2.2), we can use the well-known relation between the Christoffel symbol and the metric,

$$
\Gamma_{\rho \sigma}^{\mu}=\frac{1}{2} g^{\mu \alpha}\left(\partial_{\rho} g_{\alpha \sigma}+\partial_{\sigma} g_{\alpha \rho}-\partial_{\alpha} g_{\rho \sigma}\right),
$$

as well as the graviton definition of eq. (2.25), to obtain

$$
\left[\Phi_{\Gamma}(\gamma)\right]_{\sigma}^{\mu}=\mathcal{P} \exp \left[-\frac{\kappa}{2} \int_{\gamma} d x^{\rho}\left(\partial_{\rho} h_{\sigma}^{\mu}+\partial_{\sigma} h_{\rho}^{\mu}-\partial^{\mu} h_{\rho \sigma}+\ldots\right)\right],
$$

where the ellipsis denotes terms of higher order in $\kappa$. Now let us choose the case of a straight-line contour emanating from the origin, as would be appropriate for a fast-moving particle with momentum $p^{\mu}$ emerging from a scattering process:

$$
x^{\mu}=s p^{\mu}, \quad 0 \leq s<\infty .
$$

The first term in eq. (3.21) is a total derivative, and integrates to give a gauge-dependent artifact associated with the endpoints of the contour. It will vanish for physical processes e.g. in forming gauge-invariant amplitudes, or squaring amplitudes to form a cross-section, which involves closing Wilson line contours to make a closed loop. The remaining terms take the form

$$
-\frac{\kappa}{2} \int_{0}^{\infty} d s p^{\rho}\left(\partial_{\sigma} h_{\rho}^{\mu}-\partial^{\mu} h_{\rho \sigma}\right)=\frac{i \kappa}{2} p^{\rho} \int \frac{d^{d} k}{(2 \pi)^{d}} \int_{0}^{\infty} d s\left(k_{\sigma} \tilde{h}_{\rho}^{\mu}-k^{\mu} \tilde{h}_{\rho \sigma}\right) e^{-i s k \cdot p},
$$

where we have introduced the Fourier components of the graviton field via

$$
h_{\mu \nu}=\int \frac{d^{d} k}{(2 \pi)^{d}} \tilde{h}_{\mu \nu}(k) e^{-i k \cdot x} .
$$

Carrying out the $s$ integral in eq. (3.23) yields $^{8}$

$$
\ln \left(\Phi_{g}\right) \sim \int \frac{d^{d} k}{(2 \pi)^{d}} \tilde{h}_{\beta \rho}(k)\left[\frac{\kappa}{2} \frac{p^{\rho} k_{\alpha}\left(M^{\alpha \beta}\right)^{\mu}{ }_{\sigma}}{p \cdot k}\right],
$$

where we have written the second line in terms of the spin-1 Lorentz generators of eq. (2.18). The square bracketed factor can be recognised as the appropriate contribution to the next-to-soft theorem for emission of a graviton [65]. Its appearance in this context arises given that the spin-dependent coupling to the worldline is suppressed by a single power of the momentum of emitted radiation compared to the leading Wilson line operator of eq. (2.24). Furthermore, one may perform an analogous calculation for the gauge theory operator of eq. (3.16), finding

$$
\ln \left(\Phi_{F}\right) \sim \int \frac{d^{d} k}{(2 \pi)^{d}} \tilde{A}_{\mu}^{a}(k)\left[g \tilde{\mathbf{T}}^{a} \frac{k_{\nu} M^{\mu \nu}}{p \cdot k}\right]
$$

\footnotetext{
${ }^{8}$ The upper limit of the $s$ integral in eq. (3.23) will vanish upon careful implementation of the Feynman $i \varepsilon$ prescription.
} 
again in agreement with the appropriate next-to-soft theorem [66]. The known double copy properties of these results in the study of scattering amplitudes again corroborates the fact that the single copy of the gravitational holonomy is the operator of eq. (3.16). It is also worth noting that these generalised Wilson lines were derived before in the context of next-to-soft physics, before the next-to-soft theorems were more widely recognised [29, 67].

Having presented a variety of arguments in favour of our single copy holonomy operator, let us now note that further useful insights can be obtained by focusing on a particular class of solutions, namely the Kerr-Schild solutions in terms of which the first classical double copy was formulated [43].

\subsection{Insights from Kerr-Schild solutions}

Kerr-Schild solutions of General Relativity (GR) are those for which the metric assumes a particularly simple form, namely

$$
g_{\mu \nu}=\bar{g}_{\mu \nu}+\phi(x) k_{\mu} k_{\nu} .
$$

Here $\bar{g}_{\mu \nu}$ is a background metric, which we will take to be Minkowski throughout $\left(\bar{g}_{\mu \nu}=\eta_{\mu \nu}\right)$, albeit not necessarily in Cartesian coordinates. Furthermore, $\phi(x)$ is a scalar field and $k_{\mu}$ a 4-vector field which is both null and geodesic:

$$
\bar{g}^{\mu \nu} k_{\mu} k_{\nu}=g^{\mu \nu} k_{\mu} k_{\nu}=0, \quad k \cdot D k_{\mu}=0 .
$$

Comparing with eq. (2.25), we see that Kerr-Schild solutions have a graviton field given by

$$
h_{\mu \nu}=\phi k_{\mu} k_{\nu}
$$

This ansatz turns out to greatly simplify the Einstein equations, which then have a linear dependence on the graviton only. This allows for an infinite family of exact solutions to be obtained, which incudes e.g. known black holes. Also, the "factorised" form of the graviton (i.e. involving an outer product of a 4 -vector with itself) allows a single copy to be straightforwardly obtained. Reference [43] proved that for static solutions, the gauge field

$$
\mathbf{A}_{\mu}=A_{\mu}^{a} \mathbf{T}^{a}, \quad A_{\mu}^{a}=c^{a} \phi k_{\mu},
$$

where $c^{a}$ is an arbitrary colour vector, solves the Yang-Mills equations, which again simplify to a linear form. Consequently, the field strength tensor for the gauge field takes an abelian-like form:

$$
F_{\mu \nu}^{a}(x)=D_{\mu} A_{\nu}^{a}(x)-D_{\nu} A_{\mu}^{a}(x) .
$$

In an orthonormal basis, one has

$$
g_{\mu \nu}=\eta_{a b} e^{a}{ }_{\mu} e_{\nu}
$$

which in turn implies the following form for the Kerr-Schild vierbein:

$$
e_{\mu}^{a}=\bar{e}_{\mu}^{a}+\frac{1}{2} \phi k^{a} k_{\mu}, \quad e_{a}^{\mu}=\bar{e}_{a}{ }^{\mu}-\frac{1}{2} \phi k_{a} k^{\mu} .
$$


Here $\bar{e}^{a}{ }_{\mu}$ is the vierbein associated with the background metric in eq. (3.27), which for our purposes is the Minkowski metric $\eta_{\mu \nu}$. As we review in appendix A, the spin connection associated with this particular vierbein (subject to the additional conditions of eq. (3.28)) assumes the form

$$
\left(\omega_{\mu}\right)_{a b}=\partial_{b} e_{a \mu}-\partial_{a} e_{b \mu} .
$$

Unlike the general expression of eq. (2.11), this has the pleasing property of being linear in the vierbein. Substituting the results of eq. (3.32) (after lowering indices appropriately) yields

$$
\begin{aligned}
\left(\omega_{\mu}\right)_{a b} & =\frac{1}{2}\left[\partial_{b}\left(\phi k_{\mu} k_{a}\right)-\partial_{a}\left(\phi k_{\mu} k_{b}\right)\right] \\
& =\frac{1}{2}\left[e_{b}^{\sigma} \partial_{\sigma}\left(\phi k_{\mu} k_{a}\right)-e_{a}^{\sigma} \partial_{\sigma}\left(\phi k_{\mu} k_{b}\right)\right] .
\end{aligned}
$$

Note that due to the null property of the Kerr-Schild vectors, $k^{\mu} k_{\mu}=0$, conversion between coordinate and orthonormal bases is done simply with the background vierbein:

$$
k_{a}=e_{a}{ }^{\mu} k_{\mu}=\bar{e}_{a}{ }^{\mu} k_{\mu}-\frac{1}{2} \phi k_{a} k^{\mu} k_{\mu}=\bar{e}_{a}{ }^{\mu} k_{\mu} .
$$

Thus, the spin connection in eq. (3.35) can be written as

$$
\left(\omega_{\mu}\right)_{a b}=\frac{1}{2}\left[\bar{e}_{a}^{\nu} e_{b}^{\sigma}-\bar{e}_{b}^{\nu} e_{a}^{\sigma}\right] \partial_{\sigma}\left(\phi k_{\mu} k_{\nu}\right) .
$$

If we now write the remaining vierbeins explicitly, a great deal of simplification occurs. To see this, consider the first term in the above expression:

$$
\begin{aligned}
\bar{e}_{a}{ }^{\nu} e_{b}{ }^{\sigma} \partial_{\sigma}\left(\phi k_{\mu} k_{\nu}\right) & =\bar{e}_{a}{ }^{\nu}\left[\bar{e}_{b}{ }^{\sigma}-\frac{1}{2} \phi k_{b} k^{\sigma}\right] \partial_{\sigma}\left(\phi k_{\mu} k_{\nu}\right) \\
& =\bar{e}_{a}{ }^{\nu} \bar{e}_{b}{ }^{\sigma} \partial_{\sigma}\left(\phi k_{\mu} k_{\nu}\right)-\frac{1}{2} \phi k_{a} k_{b} k_{\mu} k^{\sigma} \partial_{\sigma} \phi
\end{aligned}
$$

In the second equality, we have expanded $\partial_{\sigma}\left(\phi k_{\mu} k_{\nu}\right)$ using the product rule, from which two of the three resulting terms vanish due to the geodesic condition $k^{\sigma} \partial_{\sigma} k_{\mu}=0$. Performing the same procedure for the second term in eq. (3.37), we find that the terms which contain only a derivative of the scalar field $\phi$ cancel, such that eq. (3.37) is simply

$$
\left(\omega_{\mu}\right)_{a b}=\frac{1}{2}\left[\bar{e}_{a}^{\nu} \bar{e}_{b}^{\sigma}-\bar{e}_{b}^{\nu} \bar{e}_{a}^{\sigma}\right] \partial_{\sigma}\left(\phi k_{\mu} k_{\nu}\right) .
$$

Finally, if we expand the spin connection in terms of the Lorentz generators, we obtain

$$
\frac{i}{2}\left(\omega_{\mu}\right)_{c d} M^{c d}=-\frac{i}{2} \partial_{\sigma}\left(\phi k_{\mu} k_{\nu}\right) M^{\nu \sigma},
$$

where we have identified the spin-1 Lorentz generators as

$$
\left(M^{\nu \sigma}\right)_{a b}=i\left[\bar{e}_{a}^{\nu} \bar{e}_{b}^{\sigma}-\bar{e}_{b}^{\nu} \bar{e}_{a}^{\sigma}\right] .
$$

Thus, we are left with a simple expression in which the exponent appearing in the Kerr-Schild gravitational holonomy operator is written directly in terms of the graviton:

$$
\oint d x^{\mu}\left(\omega_{\mu}\right)_{a b} M^{a b}=-\oint d x^{\mu} \partial_{\sigma}\left(h_{\mu \nu}\right) M^{\nu \sigma} .
$$




\begin{tabular}{|c|c|}
\hline Gauge Theory & Gravity \\
\hline $\mathcal{P} \exp \left[-g \oint_{C} d x^{\mu} \mathbf{A}_{\mu}\right]$ & $\exp \left[\frac{i \kappa}{2} \oint_{C} d s \dot{x}^{\mu} \dot{x}^{\nu} h_{\mu \nu}\right]$ \\
$\mathcal{P} \exp \left[\frac{g}{2} \oint_{C} d s \mathbf{F}_{\mu \nu} M^{\mu \nu}\right]$ & $\mathcal{P} \exp \left[-\frac{i \kappa}{2} \oint_{C} d x^{\mu}\left(\omega_{\mu}\right)_{a b} M^{a b}\right]$ \\
\hline
\end{tabular}

Table 1. Holonomy operators in gauge and gravity theories, and their single/double copies.

The Kerr-Schild single copy of eq. (3.29) implies that we should single copy eq. (3.43) by replacing

$$
\dot{x}^{\mu} \rightarrow \tilde{\mathbf{T}}^{a}, \quad k_{\mu} \rightarrow c^{a},
$$

such that one obtains

$$
\begin{aligned}
\oint d x^{\mu}\left(\omega_{\mu}\right)_{a b} M^{a b} \rightarrow-\tilde{\mathbf{T}}^{a} \oint d s \partial_{\sigma}\left(\phi k_{\nu} c^{a}\right) M^{\nu \sigma} & =-\tilde{\mathbf{T}}^{a} \oint d s \partial_{\sigma}\left(A_{\nu}^{a}\right) M^{\nu \sigma} \\
& =\frac{1}{2} \tilde{\mathbf{T}}^{a} \oint d s F_{\nu \sigma}^{a} M^{\nu \sigma} .
\end{aligned}
$$

This agrees with the conclusion reached above, namely that the single copy of the gravitational holonomy is the operator of eq. (3.16). Note that the single copy in eq. (3.44) entails replacing a spacetime index $\mu$ with an adjoint index $a$, given that kinematic information is replaced by colour degrees of freedom.

By way of summarising the results of this section, we collect all of the operators we have discussed in table 1. The gauge theory and gravity holonomies appear in the top-left and bottom-right respectively, and the gravitational Wilson line appears in the top-right. The operator in the bottom-left corner completes the square, and has not been considered before as an analogue of the gravity holonomy, due to its not having the appropriate differential geometric definition. Indeed, its role is entirely different to the gauge theory holonomy. Considering a gauge field via a connection on a principal fibre bundle, the usual holonomy describes how vectors in the internal colour space (associated with the fibres) are transported as one moves along a curve. By contrast, the single copy of the gravity holonomy instead describes how spacetime vectors are transported, thereby linking the gauge field with vectors living in the tangent space of the base manifold. Thus, the vectors being transported in each case live in different vector spaces. Nevertheless, our hope is that the single-copy holonomy operator might also prove useful in classifying properties of different Yang-Mills solutions, and we develop this notion in the following section.

\section{Results for the single copy holonomy operator}

The gravitational holonomy is useful in that it allows us to classify solutions of General Relativity (and arbitrary manifolds more generally) into qualitatively different types. In general, one expects the holonomy group of a given manifold to be the most general group acting on vectors in the tangent space, namely $\mathrm{SO}(d)$ for a $d$-dimensional orientable spacetime in Euclidean signature, or $\mathrm{SO}(1, d-1)$ for Lorentzian signature. In some cases, however, the holonomy group reduces to a subgroup, and the classification of manifolds 
based on this idea has been widely studied (see e.g. ref. [38] for the most well-known incarnation). This suggests that our single copy holonomy operator might have a similar purpose. Certainly, the operator of eq. (3.16) defines a group of transformations for a given gauge theory solution. For ease of reference, we shall refer to eq. (3.16) as the SCH operator (short for "single copy holonomy operator"), and the resulting group of transformations as the $\mathrm{SCH}$ group. It may well be that the $\mathrm{SCH}$ group reduces for certain gauge fields. There is also the interesting possibility of taking gauge theory solutions that are known single copies of particular gravity solutions, and asking if the $\mathrm{SCH}$ and holonomy groups match up! We will investigate this by considering particular solutions, of increasing complexity.

\subsection{The Schwarzschild black hole}

Arguably the simplest non-trivial gravity solution is the Schwarzschild solution. It may be sourced by a point mass $M$ sitting at the origin, and has a known Kerr-Schild form involving spherical polar coordinates $(t, r, \theta, \varphi)$, where the functions entering eq. (3.27) may be chosen as

$$
\phi(r)=\frac{M}{4 \pi r}, \quad k_{\mu}=(1,1,0,0) .
$$

We can then use eq. (3.43) to ascertain the holonomy group, for which we must choose a number of different closed contours, and see what the various elements of the holonomy group are. Let us first choose a circular orbit at constant time $t$, in the equatorial plane, parametrised by

$$
C: \quad x^{\mu}=(0,0,0, \varphi), \quad \varphi \in[0,2 \pi) .
$$

The integral appearing in the holonomy operator then reduces to

$$
\oint_{C} d \varphi \partial_{\sigma}\left(h_{\varphi \nu}\right) M^{\nu \sigma}=0,
$$

where we have used the fact that the Kerr-Schild graviton implied by eq. (4.1) has no non-zero $h_{\varphi \nu}$ components. The element of the holonomy group associated with $C$ is thus the identity element. Furthermore, spherical symmetry implies that similar constant time loops that are tilted with respect to the equatorial plane will also have a trivial holonomy.

To achieve a non-zero result, one may instead consider the curve shown in figure 1 , consisting of three segments. The first segment $C_{1}$ is parallel to the time direction, and may be parametrised by

$$
C_{1}: \quad x^{\mu}=\left(t, r_{0}, 0,0\right), \quad 0 \leq t \leq T,
$$

such that the curve is at a fixed radius $r=r_{0}$, and of total length $T$. We have also chosen fixed values $\theta=\varphi=0$. From eq. (3.43), one finds

$$
\int_{C_{1}} d x^{\mu} \partial_{\sigma}\left(h_{\mu \nu}\right) M^{\nu \sigma}=\int_{0}^{T} d t \partial_{r}\left(h_{00}\right) M^{0 r}=-\frac{M T}{4 \pi r_{0}^{2}} M^{0 r},
$$

where we have noted the only non-zero contribution after contraction of indices in the intermediate step. The remaining segments are parametrised by

$$
\begin{array}{lll}
C_{2}: & x^{\mu}=\left(t, r_{0}+T-t, 0,0\right), & T \geq t \geq T / 2, \\
C_{3}: & x^{\mu}=\left(t, r_{0}+t, 0,0\right), & \frac{T}{2} \geq t \geq 0,
\end{array}
$$




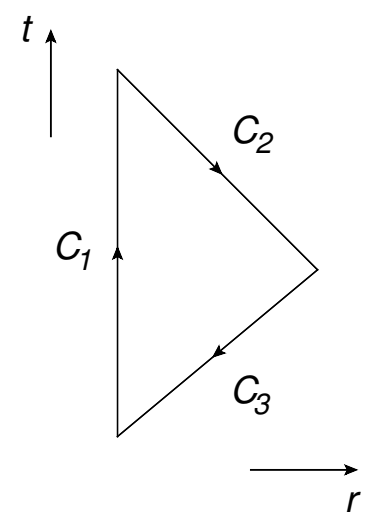

Figure 1. A loop consisting of three segments in the $(r, t)$ plane, where $C_{2}$ and $C_{3}$ are null lines. such that one finds

$$
\begin{aligned}
\int_{C_{2}} d x^{\mu} \partial_{\sigma}\left(h_{\mu \nu}\right) M^{\nu \sigma} & =\left.\int_{T}^{T / 2} d t \partial_{r}\left(h_{00}\right) M^{0 r}\right|_{r=r_{0}+T-t}+\int_{r_{0}}^{r_{0}+T / 2} d r \partial_{r}\left(h_{r 0}\right) M^{0 r} \\
\int_{C_{3}} d x^{\mu} \partial_{\sigma}\left(h_{\mu \nu}\right) M^{\nu \sigma} & =\left.\int_{T / 2}^{0} d t \partial_{r}\left(h_{00}\right) M^{0 r}\right|_{r=r_{0}+t} ^{r_{0}}+\int_{r_{0}+T / 2}^{r_{0}} d r \partial_{r}\left(h_{r 0}\right) M^{0 r}
\end{aligned}
$$

One sees that the radial components cancel. Evaluating the remaining integrals after using eq. (4.1) gives

$$
\int_{C_{2} \cup C_{3}} d x^{\mu} \partial_{\sigma}\left(h_{\mu \nu}\right) M^{\nu \sigma}=\frac{M}{2 \pi} \frac{T}{r_{0}\left(T+2 r_{0}\right)} M^{0 r},
$$

such that the total contribution from the entire loop is

$$
\oint d x^{\mu} \partial_{\sigma}\left(h_{\mu \nu}\right) M^{\nu \sigma}=\alpha M^{0 r}, \quad \alpha=-\frac{M}{4 \pi} \frac{T^{2}}{r_{0}^{2}\left(T+2 r_{0}\right)} .
$$

This constitutes an infinitesimal boost in the $(t, r)$ plane with hyperbolic angle $\alpha$. To see this, we may recall the definition of the boost generators $K_{i}$ and rotation generators $J_{i}$ in terms of the $\left\{M^{\mu \nu}\right\}$ :

$$
K_{i}=M^{0 i}, \quad J_{i}=\frac{1}{2} \epsilon_{i j k} M^{j k},
$$

in terms of which the Lorentz algebra

$$
\left[M^{\mu \nu}, M^{\rho \sigma}\right]=i\left(\eta^{\sigma \mu} M^{\rho \nu}+\eta^{\nu \sigma} M^{\mu \rho}-\eta^{\rho \mu} M^{\sigma \nu}-\eta^{\nu \rho} M^{\mu \sigma}\right)
$$

may be written as

$$
\left[J_{i}, J_{j}\right]=i \epsilon_{i j k} J_{k}, \quad\left[J_{i}, K_{j}\right]=i \epsilon_{i j k} K_{k}, \quad\left[K_{i}, K_{j}\right]=-i \epsilon_{i j k} J_{k} .
$$

Equation (4.9) is then clearly seen to contain the boost generator $K_{r}$. If one considers loops with the same $r_{0}$ but different fixed values of $\theta$ and $\varphi$, the full set of boosts associated with arbitrary directions will be obtained. This in turn implies that the holonomy group 
of the Schwarzschild spacetime is $\mathrm{SO}(1, d-1)$ : from eq. (4.12), one sees that the boosts do not close upon themselves, such that exponentiating the boost generators will produce transformations corresponding to combinations of boosts and rotations in general. Note that our conclusions are in qualitative agreement with those of e.g. ref. [68] which also considered the holonomy in Schwarzschild spacetime. Our explicit result for the boost angle differs due to having a loop with a slightly different orientation, and also the use of Kerr-Schild rather than conventional Schwarzschild coordinates.

Given this holonomy group, we may now consider the single copy, which is well-known to be an abelian-like point charge in the gauge theory [43]. We may thus consider an abelian exponent for the SCH operator:

$$
\ln \left(\Phi_{F}\right) \rightarrow i g \oint_{C} d s F_{\mu \nu} M^{\mu \nu}
$$

The only non-zero component of the field strength in this case is

$$
F_{0 r}=\frac{Q}{4 \pi r^{2}}
$$

where $Q$ is the charge. Plugging this into eq. (4.13), we see immediately that an infinitesimal boost in the $(t, r)$ plane is obtained, directly analogous to the Schwarzschild case. Thus, the $\mathrm{SCH}$ group of the point charge is $\mathrm{SO}(1, d-1)$. It is reasonable to ponder at this point whether it is always the case that the $\mathrm{SCH}$ and holonomy groups match up for gauge theory and gravity solutions related by the double copy. That this is not the case will be seen in the following example.

\subsection{Taub-NUT space}

The Taub-NUT solution, first derived in refs. [69, 70], is a non-asymptotically flat solution of GR, that has a rotational character to the gravitational field at infinity. This is due to a so-called NUT charge $N$, that is present in addition to a Schwarzschild-like mass $M$. With a suitable choice of coordinates, the metric may be written in Lorentzian signature as

$$
d s^{2}=-A(r)[d t+B(\theta) d \phi]^{2}+A^{-1}(r) d r^{2}+C(r)\left[d \theta^{2}+D(\theta) d \phi^{2}\right],
$$

where for convenience we have defined

$$
A(r)=\frac{\left(r-r_{+}\right)\left(r-r_{-}\right)}{r^{2}+N^{2}}, \quad B(\theta)=2 N \cos \theta, \quad C(r)=r^{2}+N^{2}, \quad D(\theta)=\sin ^{2} \theta
$$

and

$$
r_{ \pm}=M \pm \sqrt{M^{2}+N^{2}}
$$

The single copy of this solution was first considered in ref. [71], and relied on the fact that coordinates exist in which the Taub-NUT solution has a double Kerr-Schild form [72]. In the single copy, the mass $M$ maps to an electric charge $Q$, as is familiar from the Schwarzschild case. The NUT charge $N$, on the other hand, corresponds to a magnetic monopole in the gauge theory, such that the single copy of the full Taub-NUT solution is an electromagnetic dyon. This correspondence was considered further in refs. [6, 19], which demonstrated how 
magnetic monopoles in arbitrary non-abelian gauge theories can be mapped to NUT charge in gravity.

For our present purposes, we want to examine the relationship (if any) between the $\mathrm{SCH}$ group in gauge theory, and the holonomy in gravity. Given that we have already seen this relationship for a mass term $M$ in gravity, it is convenient to take this to zero, and thus to consider the metric associated with a pure NUT charge $N$, which maps to a magnetic monopole in gauge theory, whose magnetic field may be written as

$$
\boldsymbol{B}=\frac{\tilde{g}}{4 \pi r^{2}} \hat{\boldsymbol{r}},
$$

where $\tilde{g}$ is the magnetic charge, $r$ the spherical radius and $\hat{\boldsymbol{r}}$ a unit vector in the radial direction. Note that we have again chosen an abelian gauge group in the single copy for simplicity, but the generalisation to a non-abelian context is straightforward [6, 19]. From eq. (3.17), one sees that the only non-zero components of the electromagnetic field strength are

$$
F_{\theta \phi}=-F_{\phi \theta}=B_{r}=\frac{\tilde{g}}{4 \pi r^{2}} .
$$

Thus, the integral appearing in the $\mathrm{SCH}$ operator of eq. (3.16) reduces to

$$
\oint_{C} d s \frac{\tilde{g}}{2 \pi r} M^{\theta \phi},
$$

such that only the rotation generator in the $(\theta, \phi)$ plane is turned on. This integral is indeed non-zero in general. Perhaps the simplest case one may consider is a constant-time curve of radius $r=r_{0}$ in the equatorial plane. All factors appearing in the integrand in eq. (4.20) can then be taken outside the integral, which then simply yields the length of the curve. Taking all possible curves, the generator $M^{\theta \phi}$ will generate rotations in all possible (Cartesian) directions, but not boosts. From eq. (4.12), one sees that the rotation algebra closes upon itself. Thus, we straightforwardly obtain that the $\mathrm{SCH}$ group of the magnetic monopole is $\mathrm{SO}(3)$ in four dimensions, and is therefore reduced compared to the electric case of $\mathrm{SO}(1,3)$.

Let us now consider whether this matches up with the holonomy group of the Taub-NUT solution in gravity. Given the metric of eq. (4.15), we may choose the vierbein

$$
e^{0}=A^{\frac{1}{2}}(d t+B d \phi), \quad e^{1}=A^{-\frac{1}{2}} d r, \quad e^{2}=C^{\frac{1}{2}} d \theta, \quad e^{3}=(C D)^{\frac{1}{2}} d \phi .
$$

It will also be useful to have the inverse of these expressions:

$$
d t=A^{-\frac{1}{2}} e^{0}-B(C D)^{-\frac{1}{2}} e^{3}, \quad d r=A^{\frac{1}{2}} e^{1}, \quad d \theta=C^{-\frac{1}{2}} e^{2}, \quad d \phi=(C D)^{-\frac{1}{2}} e^{3} .
$$

The spin connection can be obtained from the torsion-free form of Cartan's structure equations,

$$
\omega_{b}^{a} \wedge e^{b}=-d e^{a},
$$

along with the metric compatibility condition

$$
\omega_{a b}=-\omega_{b a}
$$


Thus, we first calculate the exterior derivatives of the basis in eq. (4.21), and use eq. (4.22) to write the results in terms of the vielbein basis:

$$
\begin{aligned}
& d e^{0}=\left(\partial_{r} A^{\frac{1}{2}}\right) e^{1} \wedge e^{0}+\left(\partial_{\theta} B\right) C^{-1}\left(\frac{A}{D}\right)^{\frac{1}{2}} e^{2} \wedge e^{3}, \\
& d e^{1}=0, \\
& d e^{2}=\left(\partial_{r} C^{\frac{1}{2}}\right)\left(\frac{A}{C}\right)^{\frac{1}{2}} e^{1} \wedge e^{2}, \\
& d e^{3}=\left(\partial_{r} C^{\frac{1}{2}}\right)\left(\frac{A}{C}\right)^{\frac{1}{2}} e^{1} \wedge e^{3}+\left(\partial_{\theta} D^{\frac{1}{2}}\right)\left(\frac{1}{C D}\right)^{\frac{1}{2}} e^{2} \wedge e^{3} .
\end{aligned}
$$

These can now be used in the Cartan structure equations of eq. (4.23) which, along with the metric compatibility condition in eq. (4.24), yields the following non-zero components of the spin connection:

$$
\begin{aligned}
& \omega^{0}{ }_{1}=\omega_{0}^{1}=\left(\partial_{r} A^{\frac{1}{2}}\right) A^{\frac{1}{2}}(d t+B d \phi), \\
& \omega_{2}^{0}=\omega_{0}^{2}=\frac{1}{2}\left(\partial_{\theta} B\right)\left(\frac{A}{C}\right)^{\frac{1}{2}} d \phi, \\
& \omega_{3}^{0}=\omega_{0}^{3}=-\frac{1}{2}\left(\partial_{\theta} B\right)\left(\frac{A}{C D}\right)^{\frac{1}{2}} d \theta, \\
& \omega_{2}^{1}=-\omega_{1}^{2}=-\left(\partial_{r} C^{\frac{1}{2}}\right) A^{\frac{1}{2}} d \theta \\
& \omega_{3}^{1}=-\omega^{3}{ }_{1}=-\left(\partial_{r} C^{\frac{1}{2}}\right)(A D)^{\frac{1}{2}} d \phi \\
& \omega_{3}^{2}=-\omega_{2}^{3}=-\frac{1}{2}\left(\partial_{\theta} B\right) \frac{A}{C D^{\frac{1}{2}}}(d t+B d \phi)-\left(\partial_{\theta} D^{\frac{1}{2}}\right) d \phi .
\end{aligned}
$$

Now by substituting eqs. (4.16) and performing the derivatives we find for Taub-NUT:

$$
\begin{aligned}
& \omega_{1}^{0}=\omega_{0}^{1}=\frac{M\left(r^{2}-N^{2}\right)+2 N^{2} r}{\left(r^{2}+N^{2}\right)^{2}}[d t+2 N \cos \theta d \phi] \\
& \omega^{0}{ }_{2}=\omega_{0}^{2}=-\frac{N \sin \theta}{r^{2}+N^{2}} \sqrt{\left(r-r_{+}\right)\left(r-r_{-}\right)} d \phi \\
& \omega^{0}{ }_{3}=\omega^{3}{ }_{0}=\frac{N}{r^{2}+N^{2}} \sqrt{\left(r-r_{+}\right)\left(r-r_{-}\right)} d \theta \\
& \omega^{1}{ }_{2}=-\omega^{2}{ }_{1}=-\frac{r}{r^{2}+N^{2}} \sqrt{\left(r-r_{+}\right)\left(r-r_{-}\right)} d \theta \\
& \omega_{3}^{1}=-\omega_{1}^{3}=-\frac{r \sin \theta}{r^{2}+N^{2}} \sqrt{\left(r-r_{+}\right)\left(r-r_{-}\right)} d \phi \\
& \omega_{3}^{2}=-\omega_{2}{ }_{2}=\frac{N\left(r-r_{+}\right)\left(r-r_{-}\right)}{\left(r^{2}+N^{2}\right)^{2}} d t+\left[\frac{2 N^{2}\left(r-r_{+}\right)\left(r-r_{-}\right)}{\left(r^{2}+N^{2}\right)^{2}}-1\right] \cos \theta d \phi .
\end{aligned}
$$

For the single copy solution of a magnetic monopole above, we considered a loop at constant time and radius in the equatorial plane $\theta=\pi / 2$. The integral in the holonomy operator is then simply

$$
\oint d x^{\mu}\left(\omega_{\mu}\right)_{a b} M^{a b}=2 \oint d \phi\left[\left(\omega_{\phi}\right)_{02} M^{02}+\left(\omega_{\phi}\right)_{13} M^{13}\right]=4 \pi\left[\left(\omega_{\phi}\right)_{02} M^{02}+\left(\omega_{\phi}\right)_{13} M^{13}\right] .
$$


This yields a boost in the 0-2 plane and a rotation in the 1-3 plane, with coefficients $\left(\omega_{\phi}\right)_{02}$ and $\left(\omega_{\phi}\right)_{13}$ respectively, where

$$
\begin{aligned}
& \left(\omega_{\phi}\right)_{02}=\frac{N}{r^{2}+N^{2}} \sqrt{\left(r-r_{+}\right)\left(r-r_{-}\right)}, \\
& \left(\omega_{\phi}\right)_{13}=-\frac{r}{r^{2}+N^{2}} \sqrt{\left(r-r_{+}\right)\left(r-r_{-}\right)} .
\end{aligned}
$$

Note that the boost and rotation planes are mutually orthogonal, and such a transformation is conventionally referred to as a Lorentz four-screw. Furthermore, our results are in agreement with those of ref. [73], despite the different choice of vierbein adopted by that reference. Equations (4.42), (4.43) still correspond to the general Taub-NUT solution. We wish to consider the double copy of the pure magnetic monopole, i.e. a pure NUT charge, such that we may set $M \rightarrow 0$ in eqs. (4.42), (4.43). The integral of eq. (4.41) then becomes

$$
\oint d x^{\mu}\left(\omega_{\mu}\right)_{a b} M^{a b}=\frac{4 \pi \sqrt{r^{2}-N^{2}}}{r^{2}+N^{2}}\left[N M^{02}-r M^{13}\right] .
$$

We thus see that the boost generator survives even in the case of a pure NUT charge. By the arguments of the previous section, this will potentially lead to the holonomy group $\mathrm{SO}(1,3)$, unless the effect of the boost can be removed by performing a similarity transformation on all group elements. However, upon considering other loops, boosts in different Cartesian directions are generated. To see this, we may use the fact that the metric for a pure NUT charge has a single Kerr-Schild form, and thus we may use the expression of eq. (3.41) for the integrand of holonomy operator. The coefficient of the boost generators, including the measure, is then

$$
d x^{\mu} \partial_{\sigma}\left(\phi k_{\mu}\right) M^{0 \sigma}=d x^{\mu}\left[\partial_{\sigma}\left(\phi k_{\mu}\right)-\partial_{\mu}\left(\phi k_{\sigma}\right)\right] M^{0 \sigma},
$$

where we have used the fact that $k_{0}=1$ for this solution [74], and in the second term we have introduced a total derivative term that integrates to zero around a closed loop. From eq. (3.29), we may recognise the expression in the closed brackets as the field strength tensor of a gauge field that is the single copy of a Kerr-Schild graviton. One then finds

$$
\begin{aligned}
d x^{\mu} \partial_{\sigma}\left(\phi k_{\mu}\right) M^{0 \sigma} & =d x^{\mu} F_{\sigma \mu} M^{0 \sigma} \\
& =d x_{j} \epsilon_{i j k} B_{k} K_{i} \\
& =\boldsymbol{K} \cdot[d \boldsymbol{x} \times \boldsymbol{B}],
\end{aligned}
$$

where we have used eqs. (3.17), (4.10). The physical content of eq. (4.46) can be understood by considering a loop at constant time and radius, that is tilted relative to the equatorial plane, as shown in figure 2. The field of a magnetic monopole points radially outwards, whereas the tangent 3 -vector to the curve $d \boldsymbol{x}$ points into the page at the point shown. This generates a boost in the direction $\boldsymbol{k} \propto d \boldsymbol{x} \times \boldsymbol{B}$, which is easily seen to be in the increasing $\theta$ direction. Thus, the conclusion reached above for the $\theta=\pi / 2$ case, namely that there is a boost in the $(t, \theta)$ plane, turns out to be general for all such constant time loops. We can therefore conclude that boosts in all Cartesian directions will be turned on, such that the 


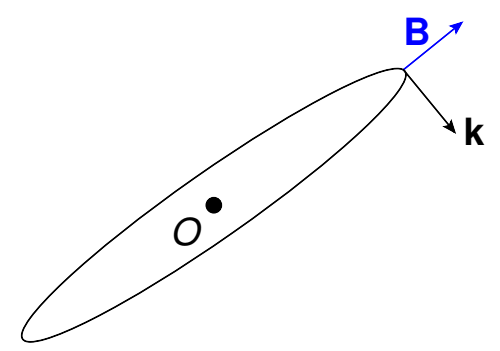

Figure 2. A closed spatial loop tilted with respect to the equatorial plane, where $O$ denotes the origin. A monopole magnetic field $\boldsymbol{B}$ generates a boost in the direction $\boldsymbol{k}$.

holonomy group of the pure NUT charge is indeed $\mathrm{SO}(1,3)$, in contrast to the $\mathrm{SCH}$ group of the magnetic monopole. Naïvely, one might have expected these groups to match up, given that there are a well-known set of analogies between electromagnetism and gravity known as gravitomagnetism. The pure NUT charge is an extremal case of the Taub-NUT solution, that is most like a magnetic monopole from a purely gravitational point of view. However, the fact that its holonomy is sensitive to gravitoelectric as well as gravitomagnetic effects is well-documented [73], and arises from the fact that spacelike hypersurfaces in the NUT solution have a nonzero extrinsic curvature. This is not the case in the single copy gauge theory, which lives in Minkowski space. ${ }^{9}$

\subsection{Self-dual solutions}

The above examples probe different types of behaviour of the SCH operator. For the Schwarzschild/point charge system, neither the SCH nor the holonomy group reduce from their general form of $\mathrm{SO}(1,3)$. For the NUT/monopole solutions, the SCH group does indeed reduce, but the holonomy does not. In this section, we demonstrate another possibility. Namely, that the SCH and holonomy groups both reduce to mutually isomorphic subgroups of $\mathrm{SO}(1,3)$. We will explicitly consider the case of self-dual solutions in gauge theory and gravity. In gravity, one may decompose the Riemann tensor into self-dual and anti-self-dual parts, given respectively by

$$
R_{\mu \nu \rho \lambda}^{ \pm}=\left(P^{ \pm}\right)^{\alpha \beta}{ }_{\mu \nu} R_{\alpha \beta \rho \lambda}
$$

where we have defined the projectors

$$
\left(P^{ \pm}\right)_{\mu \nu}^{\alpha \beta}=\frac{1}{2}\left(\delta_{\mu}^{\alpha} \delta_{\nu}^{\beta}-\delta_{\nu}^{\alpha} \delta_{\mu}^{\beta} \pm \sqrt{g} \epsilon_{\mu \nu}^{\alpha \beta}\right)
$$

with $g$ denoting the determinant of the metric. Note also that we work in Euclidean signature throughout this section only. We may use Stokes' theorem to rewrite the holonomy operator

\footnotetext{
${ }^{9}$ The fact that both electric and magnetic-like generators are turned on in gravity can also be understood in terms of the intrinsic non-linearity of GR compared to abelian gauge theory. We are grateful to the anonymous referee for raising this point.
} 
of eq. (2.19) as [31]

$$
\begin{aligned}
\Phi_{\omega} & =\mathcal{P} \exp \left(-\frac{i}{2} \iint_{\Sigma} d \Sigma^{\mu \nu} R_{c d \mu \nu} M^{c d}\right) \\
& =\mathcal{P} \exp \left(-\frac{i}{2} \iint_{\Sigma} d \Sigma^{\mu \nu}\left(R_{\rho \lambda \mu \nu}^{+}+R_{\rho \lambda \mu \nu}^{-}\right) M^{\rho \lambda}\right),
\end{aligned}
$$

where $\Sigma$ is the area bounded by the curve $C$, with area element $d \Sigma^{\mu \nu}$, and we have also converted vielbein indices to coordinate frame indices in the second line. Note that $\Sigma$ is simply connected, and therefore the holonomy group reduces to the restricted holonomy group $\operatorname{Hol}^{0}(\nabla)$, which is only equal to the full group $\mathrm{Hol}(\nabla)$ when the fundamental homotopy group $\pi_{1}$ is trivial. The second term is zero by definition for a self-dual solution, in which case the holonomy operator becomes

$$
\begin{aligned}
\Phi_{\omega} & =\mathcal{P} \exp \left(-\frac{i}{2} \iint_{\Sigma} d \Sigma^{\mu \nu}\left(P^{+}\right)_{\rho \lambda}^{\alpha \beta} R_{\alpha \beta \mu \nu} M^{\rho \lambda}\right) \\
& =\mathcal{P} \exp \left(-\frac{i}{2} \iint_{\Sigma} d \Sigma^{\mu \nu} R_{\alpha \beta \mu \nu}\left(M^{+}\right)^{\alpha \beta}\right),
\end{aligned}
$$

where we have defined two linearly independent sets of Lorentz generators via

$$
\left(M^{ \pm}\right)^{\alpha \beta}=\left(P^{ \pm}\right)_{\rho \lambda}^{\alpha \beta} M^{\rho \lambda} .
$$

This amounts to the known lie algebra isomorphism $\mathfrak{s o}(4) \simeq \mathfrak{s u}(2) \oplus \mathfrak{s u}(2)$, where each $\mathfrak{s u}(2)$ subgroup corresponds to the generators $\left(M^{ \pm}\right)^{\alpha \beta}$ respectively. It follows that for self-dual solutions, the holonomy group reduces to $\mathrm{SU}(2)$, and similar arguments can be applied to the case of anti-self-dual solutions.

The single copy of a self-dual gravity solution is also self-dual in the gauge theory [75]. One may define the (anti-)self-dual parts of the field strength as follows:

$$
F_{\mu \nu}^{ \pm}=\left(P^{ \pm}\right)_{\mu \nu}^{\alpha \beta} F_{\alpha \beta},
$$

where one uses the projectors of eq. (4.48), but where now the metric corresponds to that of Euclidean flat space (albeit potentially in a curvilinear coordinate system, so that one must keep the factor of $\sqrt{g}$ ). Self-dual solutions are defined by $F_{\mu \nu}^{-}=0$, so that the SCH operator becomes

$$
\begin{aligned}
\exp \left[i g \oint_{C} d s F_{\mu \nu}^{+} M^{\mu \nu}\right] & =\exp \left[i g \oint_{C} d s\left(P^{+}\right)_{\mu \nu}{ }^{\alpha \beta} F_{\alpha \beta} M^{\mu \nu}\right] \\
& =\exp \left[i g \oint_{C} d s F_{\alpha \beta}\left(M^{+}\right)^{\alpha \beta}\right] .
\end{aligned}
$$

Again only half of the generators are turned on, so that the SCH group reduces to $\mathrm{SU}(2)$. The self-dual sector thus provides an interesting example, in which the SCH and holonomy groups reduce, and are isomorphic. Furthermore, it is interesting to ponder whether the arguments of this section generalise to manifolds of exotic holonomy in higher dimensions, such as the well-known cases with holonomy groups $\mathrm{G}_{2}$ and $\operatorname{Spin}(7)$. It is not known how to explicitly single copy such manifolds (see e.g. ref. [76] for a related discussion), but seeking Yang-Mills solutions with a suitable SCH group might be a good place to start. 


\section{Discussion}

In this paper, we have considered the holonomy group in gravity, which consists of the group of transformations acting on vectors that have been parallel transported around a closed curve. The analogue of this in a (non)-abelian gauge theory is the Wilson loop, which has a physical interpretation in terms of the phase experienced by a scalar particle traversing a closed contour. Attempts to match up the physical properties of the holonomy and Wilson line - or to interpret the holonomy operator itself as a gravitational Wilson loop - have been made before [21-24, 26], with the conclusion that the gravitational holonomy should not be thought as the being the correct physical analogue of the gauge theory Wilson line. Indeed, a different gravitational Wilson line exists, which corresponds to the phase experienced by a scalar particle $[27-29,31] .{ }^{10}$ This begs the question of what the correct gauge theory analogue of the gravitational holonomy is. To investigate this we have used the single copy. We showed that the gravitational holonomy arises naturally in the description of a spinning particle interacting with a gravitatonal field. The single copy of this situation is well-known to be a spinning particle interacting with a gauge field. This allowed us to construct a generalised Wilson line operator in the gauge theory, which gives the phase (non-diagonal in spin space) experienced by a spin-1 test particle having an electric and magnetic dipole moment.

Having found the single copy of the holonomy operator - which we dubbed the SCH operator - we then commenced an exploration of its properties. We looked at certain special cases in which the $\mathrm{SCH}$ group reduces, which includes the case of a pure magnetic monopole, and also solutions which are self-dual. For the former, the SCH group reduces even though the gravitational holonomy of the monopole's double copy counterpart (a pure NUT charge) does not.

There are a number of avenues for further work. Firstly, one could apply the SCH operator to different Yang-Mills solutions, and see what general conclusions can be reached about their possible SCH groups. It would also be interesting to look at how to match the holonomy and its single copy in gauge and gravity theories more generally, which might help in extending the classical double copy to more complicated cases than are currently possible. Thirdly, it would be nice if the SCH operator could shed light on non-perturbative aspects of the double copy. In particular, we note that the SCH operator is matrix-valued both in colour and spin space. It thus rotates vectors both in the internal space associated with the colour degrees of freedom, and also in the tangent space of the manifold, which is associated with kinematic information. Might the single copy of the holonomy then have something to do with BCJ duality [77], which links colour and kinematics in an intriguing way?

\footnotetext{
${ }^{10}$ The transformation groups spanned by the gauge theory holonomy and the gravitational Wilson line will be different in general. Interestingly, however, they match up for the case of abelian gauge theory, given that both the abelian gauge transformations and gravitational phases correspond to the group U(1). We thank the anonymous referee for this observation.
} 


\section{Acknowledgments}

We thank Stefano De Angelis, Jung-Wook Kim, Adrian Padellaro, Malcolm Perry and Rajath Radhakrishnan for many useful discussions. We also thank Jan Plefka for correspondence, some time ago, about the use of the first-order point particle action in the double copy. DSB is happy to acknowledge the support of Pierre Andurand. This work has been supported by the UK Science and Technology Facilities Council (STFC) Consolidated Grant ST/P000754/1 "String theory, gauge theory and duality", and by the European Union Horizon 2020 research and innovation programme under the Marie Skłodowska-Curie grant agreement No. 764850 "SAGEX". RA is supported by a student scholarship from the Ministry of Education of the UAE.

\section{A Derivation of the Kerr-Schild spin connection}

In this appendix, we provide a derivation of the form of the spin connection in Kerr-Schild coordinates, as reported in eq. (3.32). The spin connection satisfies Cartan's first structure equation in the absence of torsion,

$$
d e^{a}+\omega_{c}^{a} \wedge e^{c}=0 .
$$

In tensorial language this takes the form

$$
\partial_{\mu} e^{a}{ }_{\nu}-\partial_{\nu} e_{\mu}^{a}+\left(\omega_{\mu}\right)^{a}{ }_{\nu}-\left(\omega_{\nu}\right)^{a}{ }_{\mu}=0
$$

were we have contracted the vierbein with the spin connection. Multiplying by a factor of $e_{b}^{\mu} e_{a}^{\nu}$, one finds

$$
\left(\partial_{b} e^{a}{ }_{\nu}\right) e_{a}^{\nu}-\left(\partial_{b} e^{a}{ }_{\mu}\right) e_{b}^{\mu}+\left(\omega_{b}\right)^{a}{ }_{c}-\left(\omega_{c}\right)_{b}^{a}=0 .
$$

Next, one can substitute the explicit forms of the Kerr-Schild vierbein given in eq. (3.32), and use the null condition from eq. (3.28), to obtain

$$
\partial_{b} e^{a}{ }_{c}-\partial_{c} e^{a}{ }_{b}+\left(\omega_{b}\right)^{a}{ }_{c}-\left(\omega_{c}\right)_{b}^{a}-\frac{1}{4} \phi^{2} k^{a} k^{\mu}\left[k_{c} \partial_{b} k_{\mu}-k_{b} \partial_{c} k_{\mu}\right]=0 .
$$

Upon lowering the index $a$, one may cyclically permute the indices $(a, b, c)$ and consider the combination $(a, b, c)-(b, c, a)-(c, a, b)=0$, which yields

$$
\left(\omega_{\mu}\right)_{b c}=\left(\omega_{a}\right)_{b c} e_{\mu}^{a}=\left(\partial_{c} e_{a b}-\partial_{b} e_{a c}\right) e^{a}{ }_{\mu}+\frac{1}{4} \phi^{2} k_{\mu} k^{\nu}\left(k_{c} \partial_{b} k_{\nu}-k_{b} \partial_{c} k_{\nu}\right),
$$

where we have also multiplied the entire equation by $e^{a}{ }_{\mu}$ to turn $a$ into a spacetime index. One may again use eqs. (3.32), (3.28) for the vierbein in the first term, after which cancellations occur, leading to

$$
\left(\omega_{\mu}\right)_{a b}=\partial_{b} e_{a \mu}-\partial_{a} e_{b \mu} .
$$

This agrees with a similar result in ref. [78], and can also be obtained by plugging the Kerr-Schild vierbein of eq. (3.32) into eq. (2.11). 
Open Access. This article is distributed under the terms of the Creative Commons Attribution License (CC-BY 4.0), which permits any use, distribution and reproduction in any medium, provided the original author(s) and source are credited.

\section{References}

[1] Z. Bern, J.J.M. Carrasco and H. Johansson, Perturbative Quantum Gravity as a Double Copy of Gauge Theory, Phys. Rev. Lett. 105 (2010) 061602 [arXiv:1004.0476] [InSPIRE].

[2] Z. Bern, T. Dennen, Y.-t. Huang and M. Kiermaier, Gravity as the Square of Gauge Theory, Phys. Rev. D 82 (2010) 065003 [arXiv: 1004.0693] [InSPIRE].

[3] C.D. White, Exact solutions for the biadjoint scalar field, Phys. Lett. B 763 (2016) 365 [arXiv: 1606.04724] [INSPIRE].

[4] P.-J. De Smet and C.D. White, Extended solutions for the biadjoint scalar field, Phys. Lett. B 775 (2017) 163 [arXiv:1708.01103] [INSPIRE].

[5] N. Bahjat-Abbas, R. Stark-Muchão and C.D. White, Biadjoint wires, Phys. Lett. B 788 (2019) 274 [arXiv: 1810.08118 ] [INSPIRE].

[6] N. Bahjat-Abbas, R. Stark-Muchão and C.D. White, Monopoles, shockwaves and the classical double copy, JHEP 04 (2020) 102 [arXiv:2001.09918] [INSPIRE].

[7] D.S. Berman, K. Kim and K. Lee, The classical double copy for M-theory from a Kerr-Schild ansatz for exceptional field theory, JHEP 04 (2021) 071 [arXiv: 2010.08255] [INSPIRE].

[8] R. Monteiro and D. O'Connell, The Kinematic Algebra From the Self-Dual Sector, JHEP 07 (2011) 007 [arXiv: 1105.2565] [INSPIRE].

[9] L. Borsten, B. Jurčo, H. Kim, T. Macrelli, C. Sämann and M. Wolf, Double Copy from Homotopy Algebras, Fortsch. Phys. 69 (2021) 2100075 [arXiv:2102.11390] [InSPIRE].

[10] E. Chacón, H. García-Compeán, A. Luna, R. Monteiro and C.D. White, New heavenly double copies, JHEP 03 (2021) 247 [arXiv:2008.09603] [INSPIRE].

[11] C.D. White, Twistorial Foundation for the Classical Double Copy, Phys. Rev. Lett. 126 (2021) 061602 [arXiv: 2012.02479] [INSPIRE].

[12] E. Chacón, S. Nagy and C.D. White, The Weyl double copy from twistor space, JHEP 05 (2021) 2239 [arXiv: 2103.16441] [INSPIRE].

[13] K. Farnsworth, M.L. Graesser and G. Herczeg, Twistor Space Origins of the Newman-Penrose Map, arXiv:2104.09525 [INSPIRE].

[14] R. Alawadhi, D.S. Berman, B. Spence and D. Peinador Veiga, S-duality and the double copy, JHEP 03 (2020) 059 [arXiv: 1911.06797] [INSPIRE].

[15] Y.-T. Huang, U. Kol and D. O'Connell, Double copy of electric-magnetic duality, Phys. Rev. D 102 (2020) 046005 [arXiv:1911.06318] [INSPIRE].

[16] N. Moynihan and J. Murugan, On-Shell Electric-Magnetic Duality and the Dual Graviton, arXiv:2002.11085 [INSPIRE].

[17] J.-W. Kim and M. Shim, Gravitational Dyonic Amplitude at One-Loop and its Inconsistency with the Classical Impulse, JHEP 02 (2021) 217 [arXiv:2010.14347] [INSPIRE].

[18] D.S. Berman, E. Chacón, A. Luna and C.D. White, The self-dual classical double copy, and the Eguchi-Hanson instanton, JHEP 01 (2019) 107 [arXiv: 1809.04063] [INSPIRE]. 
[19] L. Alfonsi, C.D. White and S. Wikeley, Topology and Wilson lines: global aspects of the double copy, JHEP 07 (2020) 091 [arXiv: 2004.07181] [INSPIRE].

[20] S.S. Wani, T.S. Tsun and M. Faizal, Dualized Gravity beyond Linear Approximation, arXiv:2103.02444 [INSPIRE].

[21] G. Modanese, Geodesic round trips by parallel transport in quantum gravity, Phys. Rev. D 47 (1993) 502 [INSPIRE].

[22] G. Modanese, Wilson loops in four-dimensional quantum gravity, Phys. Rev. D 49 (1994) 6534 [hep-th/9307148] [INSPIRE].

[23] H.W. Hamber and R.M. Williams, Gravitational Wilson loop and large scale curvature, Phys. Rev. D 76 (2007) 084008 [arXiv:0706.2342] [INSPIRE].

[24] H.W. Hamber and R.M. Williams, Gravitational Wilson Loop in Discrete Quantum Gravity, Phys. Rev. D 81 (2010) 084048 [arXiv:0907.2652] [INSPIRE].

[25] S. Mandelstam, Quantization of the gravitational field, Annals Phys. 19 (1962) 25 [inSPIRE].

[26] A. Brandhuber, P. Heslop, A. Nasti, B. Spence and G. Travaglini, Four-point Amplitudes in $N=8$ Supergravity and Wilson Loops, Nucl. Phys. B 807 (2009) 290 [arXiv:0805.2763] [INSPIRE].

[27] H.W. Hamber and R.M. Williams, Newtonian potential in quantum Regge gravity, Nucl. Phys. B 435 (1995) 361 [hep-th/9406163] [INSPIRE].

[28] S.G. Naculich and H.J. Schnitzer, Eikonal methods applied to gravitational scattering amplitudes, JHEP 05 (2011) 087 [arXiv:1101.1524] [INSPIRE].

[29] C.D. White, Factorization Properties of Soft Graviton Amplitudes, JHEP 05 (2011) 060 [arXiv:1103.2981] [INSPIRE].

[30] D.J. Miller and C.D. White, The Gravitational cusp anomalous dimension from AdS space, Phys. Rev. D 85 (2012) 104034 [arXiv:1201.2358] [InSPIRE].

[31] J.S. Dowker and J.A. Roche, The Gravitational analogues of magnetic monopoles, Proc. Phys. Soc. 92 (1967) 1 [INSPIRE].

[32] S. Melville, S.G. Naculich, H.J. Schnitzer and C.D. White, Wilson line approach to gravity in the high energy limit, Phys. Rev. D 89 (2014) 025009 [arXiv:1306.6019] [INSPIRE].

[33] A. Luna, S. Melville, S.G. Naculich and C.D. White, Next-to-soft corrections to high energy scattering in QCD and gravity, JHEP 01 (2017) 052 [arXiv:1611.02172] [INSPIRE].

[34] S. Oxburgh and C.D. White, BCJ duality and the double copy in the soft limit, JHEP 02 (2013) 127 [arXiv:1210.1110] [INSPIRE].

[35] A. Sabio Vera, E. Serna Campillo and M.A. Vazquez-Mozo, Color-Kinematics Duality and the Regge Limit of Inelastic Amplitudes, JHEP 04 (2013) 086 [arXiv:1212.5103] [INSPIRE].

[36] H. Johansson, A. Sabio Vera, E. Serna Campillo and M.A. Vazquez-Mozo, Color-kinematics duality and dimensional reduction for graviton emission in Regge limit, in International Workshop on Low X Physics, (2013) [arXiv:1310.1680] [INSPIRE].

[37] J. Plefka, J. Steinhoff and W. Wormsbecher, Effective action of dilaton gravity as the classical double copy of Yang-Mills theory, Phys. Rev. D 99 (2019) 024021 [arXiv:1807.09859] [INSPIRE]. 
[38] M. Berger, Sur les groupes d'holonomie homogènes de variétés à connexion affine et des variétés riemanniennes, Bull. Soc. Math. Fr. 83 (1955) 279.

[39] A.S. Galaev, Holonomy groups of Lorentzian manifolds, Russ. Math. Surveys 70 (2015) 249 [arXiv: 1611.01551] [INSPIRE].

[40] A.L. Besse, Einstein Manifolds, Springer-Verlag, Berlin, Heidelberg, New York (1987).

[41] S.M. Carroll, Spacetime and Geometry, Cambridge University Press (2019).

[42] I.A. Korchemskaya and G.P. Korchemsky, High-energy scattering in QCD and cross singularities of Wilson loops, Nucl. Phys. B 437 (1995) 127 [hep-ph/9409446] [InSPIRE].

[43] R. Monteiro, D. O'Connell and C.D. White, Black holes and the double copy, JHEP 12 (2014) 056 [arXiv: 1410.0239] [INSPIRE].

[44] M. Levi, Effective Field Theories of Post-Newtonian Gravity: A comprehensive review, Rept. Prog. Phys. 83 (2020) 075901 [arXiv: 1807.01699] [InSPIRE].

[45] A.J. Hanson and T. Regge, The Relativistic Spherical Top, Annals Phys. 87 (1974) 498 [INSPIRE].

[46] I. Bailey and W. Israel, Lagrangian Dynamics of Spinning Particles and Polarized Media in General Relativity, Commun. Math. Phys. 42 (1975) 65 [InSPIRE].

[47] W.G. Dixon, Dynamics of extended bodies in general relativity. I. Momentum and angular momentum, Proc. Roy. Soc. Lond. A 314 (1970) 499 [INSPIRE].

[48] A. Papapetrou, Spinning test particles in general relativity. 1, Proc. Roy. Soc. Lond. A 209 (1951) 248 [INSPIRE].

[49] J. Vines, D. Kunst, J. Steinhoff and T. Hinderer, Canonical Hamiltonian for an extended test body in curved spacetime: To quadratic order in spin, Phys. Rev. D 93 (2016) 103008 [Erratum ibid. 104 (2021) 029902] [arXiv: 1601.07529] [INSPIRE].

[50] J. Li and S.G. Prabhu, Gravitational radiation from the classical spinning double copy, Phys. Rev. D 97 (2018) 105019 [arXiv: 1803.02405] [INSPIRE].

[51] W.D. Goldberger, J. Li and S.G. Prabhu, Spinning particles, axion radiation, and the classical double copy, Phys. Rev. D 97 (2018) 105018 [arXiv:1712.09250] [INSPIRE].

[52] M.-Z. Chung, Y.-T. Huang, J.-W. Kim and S. Lee, The simplest massive S-matrix: from minimal coupling to Black Holes, JHEP 04 (2019) 156 [arXiv: 1812.08752] [INSPIRE].

[53] M.-Z. Chung, Y.-T. Huang and J.-W. Kim, Kerr-Newman stress-tensor from minimal coupling, JHEP 12 (2020) 103 [arXiv:1911.12775] [InSPIRE].

[54] N. Arkani-Hamed, Y.-t. Huang and D. O'Connell, Kerr black holes as elementary particles, JHEP 01 (2020) 046 [arXiv: 1906.10100] [INSPIRE].

[55] W.T. Emond, Y.-T. Huang, U. Kol, N. Moynihan and D. O'Connell, Amplitudes from Coulomb to Kerr-Taub-NUT, arXiv:2010.07861 [INSPIRE].

[56] A. Guevara, B. Maybee, A. Ochirov, D. O'connell and J. Vines, A worldsheet for Kerr, JHEP 03 (2021) 201 [arXiv:2012.11570] [INSPIRE].

[57] A. Guevara, A. Ochirov and J. Vines, Scattering of Spinning Black Holes from Exponentiated Soft Factors, JHEP 09 (2019) 056 [arXiv:1812.06895] [INSPIRE].

[58] A. Guevara, A. Ochirov and J. Vines, Black-hole scattering with general spin directions from minimal-coupling amplitudes, Phys. Rev. D 100 (2019) 104024 [arXiv:1906.10071] [INSPIRE]. 
[59] Y.F. Bautista and A. Guevara, From Scattering Amplitudes to Classical Physics: Universality, Double Copy and Soft Theorems, arXiv:1903.12419 [INSPIRE].

[60] S. Weinberg, Infrared photons and gravitons, Phys. Rev. 140 (1965) B516 [InSPIRE].

[61] D.J. Gross and R. Jackiw, Low-Energy Theorem for Graviton Scattering, Phys. Rev. 166 (1968) 1287 [INSPIRE].

[62] F.E. Low, Bremsstrahlung of very low-energy quanta in elementary particle collisions, Phys. Rev. 110 (1958) 974 [INSPIRE].

[63] T.H. Burnett and N.M. Kroll, Extension of the low soft photon theorem, Phys. Rev. Lett. 20 (1968) 86 [INSPIRE].

[64] V. Del Duca, High-energy Bremsstrahlung Theorems for Soft Photons, Nucl. Phys. B 345 (1990) 369 [INSPIRE].

[65] F. Cachazo and A. Strominger, Evidence for a New Soft Graviton Theorem, arXiv:1404.4091 [INSPIRE].

[66] E. Casali, Soft sub-leading divergences in Yang-Mills amplitudes, JHEP 08 (2014) 077 [arXiv: 1404.5551] [INSPIRE].

[67] E. Laenen, G. Stavenga and C.D. White, Path integral approach to eikonal and next-to-eikonal exponentiation, JHEP 03 (2009) 054 [arXiv:0811.2067] [INSPIRE].

[68] T. Rothman, G.F.R. Ellis and J. Murugan, Holonomy in the Schwarzschild-Droste geometry, Class. Quant. Grav. 18 (2001) 1217 [gr-qc/0008070] [INSPIRE].

[69] A.H. Taub, Empty space-times admitting a three parameter group of motions, Annals Math. 53 (1951) 472 [INSPIRE].

[70] E. Newman, L. Tamburino and T. Unti, Empty space generalization of the Schwarzschild metric, J. Math. Phys. 4 (1963) 915 [inSPIRE].

[71] A. Luna, R. Monteiro, D. O'Connell and C.D. White, The classical double copy for Taub-NUT spacetime, Phys. Lett. B $\mathbf{7 5 0}$ (2015) 272 [arXiv:1507.01869] [InSPIRE].

[72] Z.W. Chong, G.W. Gibbons, H. Lü and C.N. Pope, Separability and Killing tensors in Kerr-Taub-NUT-de Sitter metrics in higher dimensions, Phys. Lett. B 609 (2005) 124 [hep-th/0405061] [INSPIRE].

[73] D. Bini, C. Cherubini and R.T. Jantzen, Circular holonomy in the Taub NUT space-time, Class. Quant. Grav. 19 (2002) 5481 [gr-qc/0210003] [INSPIRE].

[74] A. Luna, The double copy and classical solutions, Ph.D. Thesis, University of Glasgow (2018) [http://theses.gla.ac.uk/id/eprint/8716].

[75] R. Monteiro and D. O'Connell, The Kinematic Algebras from the Scattering Equations, JHEP 03 (2014) 110 [arXiv:1311.1151] [INSPIRE].

[76] R. Alawadhi, D.S. Berman and B. Spence, Weyl doubling, JHEP 09 (2020) 127 [arXiv: 2007.03264] [INSPIRE].

[77] Z. Bern, J.J.M. Carrasco and H. Johansson, New Relations for Gauge-Theory Amplitudes, Phys. Rev. D 78 (2008) 085011 [arXiv:0805. 3993] [InSPIRE].

[78] A. Chakrabarti and D.H. Tchrakian, Antisymmetrized 2p forms generalizing curvature two forms and a corresponding $p$ hierarchy of Schwarzschild type metrics in dimensions $d>2 p+1$, Adv. Theor. Math. Phys. 3 (1999) 791 [hep-th/9908128] [INSPIRE]. 